\title{
Directional dichroism in the paramagnetic state of multiferroics: A case study of infrared light absorption in $\mathrm{Sr}_{2} \mathrm{CoSi}_{2} \mathrm{O}_{7}$ at high temperatures
}

\author{
J. Viirok, ${ }^{1}$ U. Nagel, ${ }^{1}$ T. Rõõm, ${ }^{1}$ D. G. Farkas, ${ }^{2}$ P. Balla, ${ }^{2,3}$ D. Szaller,${ }^{2,4}$ V. Kocsis,${ }^{2,5}$ Y. Tokunaga,${ }^{5,6}$ Y. Taguchi, ${ }^{5}$ \\ Y. Tokura, ${ }^{5,7}$ B. Bernáth, ${ }^{8}$ D. L. Kamenskyi, ${ }^{8}$ I. Kézsmárki, ${ }^{2,9}$ S. Bordács, ${ }^{2,10}$ and K. Penc ${ }^{2,3}$ \\ ${ }^{1}$ National Institute of Chemical Physics and Biophysics, Akadeemia tee 23, 12618 Tallinn, Estonia \\ ${ }^{2}$ Department of Physics, Budapest University of Technology and Economics and MTA-BME Lendület Magneto-optical \\ Spectroscopy Research Group, 1111 Budapest, Hungary \\ ${ }^{3}$ Institute for Solid State Physics and Optics, Wigner Research Centre for Physics, Hungarian Academy of Sciences, \\ PO Box. 49, H-1525 Budapest, Hungary \\ ${ }^{4}$ Institute of Solid State Physics, Vienna University of Technology, 1040 Vienna, Austria \\ ${ }^{5}$ RIKEN Center for Emergent Matter Science (CEMS), Wako, Saitama 351-0198, Japan \\ ${ }^{6}$ Department of Advanced Materials Science, University of Tokyo, Kashiwa, Chiba 277-8561, Japan \\ ${ }^{7}$ Department of Applied Physics, University of Tokyo, Bunkyo, Tokyo 113-8656, Japan \\ ${ }^{8}$ High Field Magnet Laboratory (HFML-EMFL), Radboud University, Toernooiveld 7, 6525 ED Nijmegen, The Netherlands \\ ${ }^{9}$ Experimental Physics V, Center for Electronic Correlations and Magnetism, Institute of Physics, \\ University of Augsburg, 86159 Augsburg, Germany \\ ${ }^{10}$ Hungarian Academy of Sciences, Premium Postdoctor Program, 1051 Budapest, Hungary
}

(Received 28 September 2018; published 9 January 2019)

\begin{abstract}
The coexisting magnetic and ferroelectric orders in multiferroic materials give rise to a handful of novel magnetoelectric phenomena, such as the absorption difference for the opposite propagation directions of light called the nonreciprocal directional dichroism (NDD). Usually, these effects are restricted to low temperature, where the multiferroic phase develops. In this paper, we report the observation of NDD in the paramagnetic phase of $\mathrm{Sr}_{2} \mathrm{CoSi}_{2} \mathrm{O}_{7}$ up to temperatures more than 10 times higher than its Néel temperature $(7 \mathrm{~K}$ ) and in fields up to $30 \mathrm{~T}$. The magnetically induced polarization and NDD in the disordered paramagnetic phase is readily explained by the single-ion spin-dependent hybridization mechanism, which does not necessitate correlation effects between magnetic ions. The $\mathrm{Sr}_{2} \mathrm{CoSi}_{2} \mathrm{O}_{7}$ provides an ideal system for a theoretical case study, demonstrating the concept of magnetoelectric spin excitations in a paramagnet via analytical as well as numerical approaches. We applied exact diagonalization of a spin cluster to map out the temperature and field dependence of the spin excitations, as well as symmetry arguments of the single ion and lattice problem to get the spectrum and selection rules.
\end{abstract}

DOI: 10.1103/PhysRevB.99.014410

\section{INTRODUCTION}

Nonreciprocal directional dichroism (NDD) is the property of a material to have different absorption coefficients for light propagation directions $\pm \mathbf{k}$ along and opposite to a given direction in the crystal [1]. Although NDD was observed a long time ago for the exciton transitions of the polar semiconductor CdS [2], it was recognized as a general magnetooptical phenomenon of noncentrosymmetric materials only by the seminal works of Rikken and his co-workers $[3,4]$. The two basic cases of NDD were identified as the magnetochiral dichroism [3] (MChD) and the toroidal dichroism. In the case of MChD the absorption coefficient is different for light propagation along and opposite the magnetization of a chiral magnet $\mathbf{k} \| \mathbf{M}$ [4]. In the case of toroidal dichroism NDD appears for propagation along and opposite $\mathbf{k} \| \mathbf{P} \times \mathbf{M}$, where $\mathbf{P}$ is the ferroelectric polarization of the material. In general, NDD can be finite only when both the spatial inversion and time-reversal symmetries are broken, as these symmetry operations interconnect the light beams propagating in opposite directions. Following the same principle, a recent study rigorously classified the magnetic point groups compatible with NDD [5]. It predicted that beside magnetochiral and toroidal dichroism NDD can arise in previously unclassified cases, which cannot be classified by a static vector quantity, such as the magnetization or the toroidal moment.

Magnetoelectric (ME) multiferroics with coexisting ferroelectric and magnetic orders naturally have the low-symmetry ground states exhibiting magnetochiral or toroidal dichroism, and indeed gigantic NDD was found in their collective excitations, typically in the $\mathrm{GHz}-\mathrm{THz}$ range [6-12]. At these low frequencies, where the electromagnetic radiation is uniform on the scale of the magnetic unit cell, NDD can solely originate from the coupled dynamics of the magnetization and the electric polarization, the dynamic ME effect. When light beams travel in a ME material, for a beam propagating in one direction the oscillating magnetization generated by the electric field of the light can enhance the conventional magnetization component, induced by the magnetic field of light, whereas these two terms interfere destructively for the counterpropagating beam since the relative phase of the electric and magnetic fields of light changes by $\pi$ when reversing the propagation direction. The direct connection 


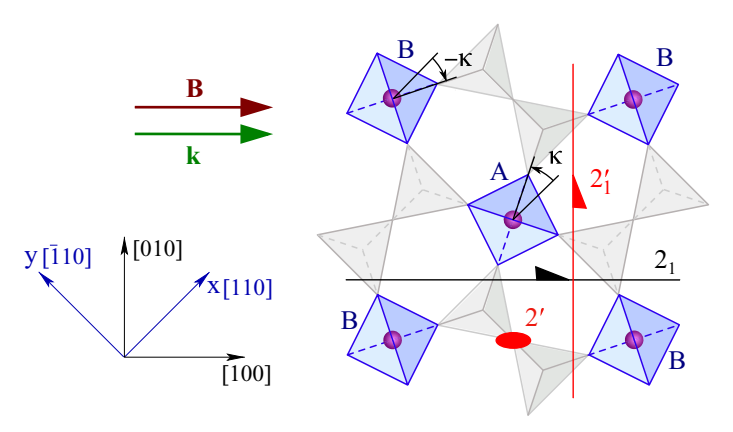

FIG. 1. Schematic illustration of the $\mathrm{Sr}_{2} \mathrm{CoSi}_{2} \mathrm{O}_{7}$ crystal structure, space group $P \overline{4} 2_{1} m$, and the coordinate axes $\mathbf{x} \|$ [110] and $\mathbf{y} \|$ [110]. Purple circles denote the $S=\frac{3}{2} \mathrm{Co}^{2+}$ ions in the centers of the $\mathrm{O}^{2-}$ tetrahedra (shown in blue). The compound is built up by layers of $\mathrm{CoO}_{4}$ tetrahedra and $\mathrm{Si}_{2} \mathrm{O}_{7}$ units (gray), which are separated by $\mathrm{Sr}$ ions. For clarity, only a single layer is shown. $\pm \kappa$ is the rotation angle of tetrahedra about the [001] axis away from [110]. A and B denote the two types of tetrahedra with $+\kappa$ and $-\kappa$ tilt. In the experiment, the $\mathrm{THz}$ light propagates along the direction of the magnetic field $\mathbf{k}\|\mathbf{B}\|[100]$. The magnetic field breaks the time-reversal symmetry and for this field direction the remaining unitary symmetry operation is the $2_{1}$ screw axis (black half-arrow). The antiunitary operations including the time reversal are the $2^{\prime}$ twofold rotation about the [001] axis (red ellipse) and the $2_{1}^{\prime}$ screw axis (red half arrow).

between the NDD and the ME effect allows the calculation of the direct current (dc) ME coefficient from the NDD spectrum using the sum rule [13]. Thus, the spectroscopic information about the ME resonances can promote the synthesis of new multiferroics with large ME coefficient.

One-way transparency is an extreme case of gigantic NDD when the light is absorbed only in one direction but not for the opposite propagation direction. Since no physical law prohibits the one-way transparency, an efficient one-way light guide can be realized. Furthermore, the transparent direction can be switched with applied magnetic [6] and possibly with electric field $[14,15]$. Some of the multiferroics, e.g., melilites $\mathrm{Ba}_{2} \mathrm{CoGe}_{2} \mathrm{O}_{7}, \mathrm{Ca}_{2} \mathrm{CoSi}_{2} \mathrm{O}_{7}$, and $\mathrm{Sr}_{2} \mathrm{CoSi}_{2} \mathrm{O}_{7}$, are not far from the ideal realization of one-way transparency [10]. In principle, these materials can find applications in photonics as diodes for $\mathrm{THz}$ radiation or directional light switches [12]. However, the multiferroic order possessing large NDD usually develops well below room temperature in the known materials, with the exception of the roomtemperature multiferroic $\mathrm{BiFeO}_{3}$ [12], rendering their use in device applications impractical.

Here, we suggest an alternative way of achieving NDD by demonstrating that the spin excitations in $\mathrm{Sr}_{2} \mathrm{CoSi}_{2} \mathrm{O}_{7}$ show NDD well above the antiferromagnetic ordering temperature $T_{\mathrm{N}}=7 \mathrm{~K}$. The crystal structure of melilites (see Fig. 1) lacks the inversion symmetry. Applying magnetic field, the time-reversal symmetry will be broken in the paramagnetic phase and the necessary conditions for NDD are then fulfilled. Akaki et al. $[16,17]$ demonstrated that in $\mathrm{Sr}_{2} \mathrm{CoSi}_{2} \mathrm{O}_{7}$ the $\mathrm{dc} \mathrm{ME}$ effect persists in the paramagnetic regime, where the field-induced polarization scales with the square of the magnetization. Since the dc ME susceptibility is related to NDD by the ME sum rule [13], we expect that NDD appears also in the paramagnetic phase of $\mathrm{Sr}_{2} \mathrm{CoSi}_{2} \mathrm{O}_{7}$.
In this paper, motivated by the discovery of the hightemperature dc ME effect in $\mathrm{Sr}_{2} \mathrm{CoSi}_{2} \mathrm{O}_{7}$, we study the NDD in the paramagnetic phase. We measured the $\mathrm{THz}$ absorption spectra in magnetic fields up to $30 \mathrm{~T}$ over a broad range of temperatures below and well above $T_{\mathrm{N}}$. The magnetic field was applied along the [100] direction, which induces magnetization parallel to the field. The point group of the $\mathrm{Sr}_{2} \mathrm{CoSi}_{2} \mathrm{O}_{7}, \overline{4} 2 m 1^{\prime}$ in the paramagnetic state, is then reduced to the magnetic point group $22^{\prime} 2^{\prime}$ [7]. In this chiral symmetry, $\mathrm{MChD}$ is expected to emerge for light propagation along the magnetic field, i.e., in the Faraday geometry. Indeed, our experiments show that the spectra are markedly different in positive and negative magnetic field, which is the hallmark of MChD. Using exact diagonalization we reproduced the magnetic field and temperature dependence of the $\mathrm{MChD}$ signal. To interpret the numerical results, a single-site analytic model was developed which shows that the finite NDD arises if all three are present: magnetic field, spin anisotropy, and ME coupling.

Recently, Yu et al. studied spin excitations of a polar ferrimagnet $\mathrm{FeZnMo}_{3} \mathrm{O}_{8}$ in Ref. [18]. They demonstrated that the toroidal dichroism can be realized in the paramagnetic phase when the light propagates along the cross product of the built-in polarization and the external magnetic-field-induced magnetization $\mathbf{k} \| \mathbf{P} \times \mathbf{M}$. In contrast, here we study $\mathrm{MChD}$, a different form of NDD, and develop microscopic spin models to understand NDD in the paramagnetic phase.

The paper is organized as follows. After description of the experimental methods in Sec. II, the experimental results are presented in Sec. III. To understand the observed spin excitations and NDD, first, a spin Hamiltonian and spin-induced polarization of $\mathrm{Sr}_{2} \mathrm{CoSi}_{2} \mathrm{O}_{7}$ are introduced in Sec. IV. The Hamiltonian is numerically diagonalized for a small cluster and the eigenstates are used to calculate magnetic and ME susceptibilities in Sec. V. Second, using a single-ion model in Sec. VI the results of the exact diagonalization are interpreted. Third, the selection rules found for the single-ion case are generalized for the lattice model in Sec. VII. In addition, in this section the effects of the exchange interaction in leading order of perturbation theory are analyzed. Finally, the main experimental and theoretical results are summarized in Sec. VIII.

\section{EXPERIMENTAL METHODS}

The $\mathrm{Sr}_{2} \mathrm{CoSi}_{2} \mathrm{O}_{7}$ crystals were grown by the floating-zone method. First, $\mathrm{SrCO}_{3}, \mathrm{Co}_{3} \mathrm{O}_{4}$, and dehydrated $\mathrm{SiO}_{2}$ were mixed in stoichiometric amount and sintered for $120 \mathrm{~h}$ at $1200^{\circ} \mathrm{C}$ in air with one intermediate regrinding. The resulting product was pressed into a rod shape and resintered for $60 \mathrm{~h}$ under the same conditions as before. The polycrystalline rod was melted into a single-crystal ingot in a halogen-incandescent lamp floating-zone apparatus (SCN35HD, NEC).

Samples for $\mathrm{THz}$ spectroscopy were disk-shaped single crystals with a diameter of $4 \mathrm{~mm}$ and thicknesses of $d=0.2$ and $0.5 \mathrm{~mm}$ in the [100] direction. The external magnetic field $\mathbf{B}$ and the light propagation $\mathbf{k}$ were both in the [100] direction $\mathbf{k}\|\mathbf{B}\|[100]$. The $\mathrm{THz}$ radiation was polarized by a wire-grid polarizer deposited on a dielectric film placed 
a few millimeters away from the sample in the incident light beam. The transmitted intensity was measured with a Martin-Puplett-type interferometer (SPS200, Sciencetech, Inc., Ontario, Canada) and $0.3 \mathrm{~K}$ composite silicon bolometer (Infrared Laboratories) in magnetic fields up to $17 \mathrm{~T}$ for both positive and negative fields in the temperature range between 3 and $100 \mathrm{~K}$. Measurements above $17 \mathrm{~T}$ were performed with a Genzel-type interferometer (Bruker 113v) and a $1.6 \mathrm{~K}$ composite Si bolometer (Infrared Laboratories).

The absorption coefficient $\alpha^{ \pm}$for magnetic fields $\pm B$ was calculated as

$$
\alpha^{ \pm}=-\frac{1}{d} \ln \frac{I( \pm B, T)}{I\left(0 \mathrm{~T}, T_{\mathrm{ref}}\right)},
$$

where $I( \pm B, T)$ is the transmitted intensity in magnetic field $B$ at temperature $T$ and $I\left(0 \mathrm{~T}, T_{\text {ref }}\right)$ is the transmitted intensity in zero magnetic field at temperature $T_{\text {ref }}$. For the magnetic field ratios $T=T_{\text {ref }}$, and the relative absorption spectra are noted as $\alpha_{\mathrm{B}}^{ \pm}$. If $B$ is constant and $T$ is varied, the ratio is denoted by $\alpha_{T}^{ \pm}$. $\alpha^{ \pm}$was determined for two polarizations of $\mathrm{THz}$ radiation $\mathbf{E}_{\omega} \|[010]$ and $\mathbf{E}_{\omega} \|[001]$.

The NDD was detected by changing the direction of the magnetic field from $+B$ to $-B$, i.e., from $\mathbf{B} \uparrow \uparrow \mathbf{k}$ to $\mathbf{B} \downarrow \uparrow \mathbf{k}$.
Due to the twofold rotation symmetry along the [001] axis, the reversal of $\mathbf{B}$ is equivalent to the reversal of $\mathbf{k}$.

\section{EXPERIMENTAL RESULTS}

Figures 2(a) and 2(b) show the temperature dependence of $\alpha_{T}^{ \pm}$between 3 and $100 \mathrm{~K}$ in two polarizations of the $\mathrm{THz}$ radiation in magnetic field $\pm 14 \mathrm{~T}$. Below $7 \mathrm{~K}$, in the magnetically ordered phase, the spectrum is dominated by three resonances as 18,28 , and $32 \mathrm{~cm}^{-1}$. Since the resonance frequencies of the spin-wave modes are located at almost the same position [10] in $\mathrm{Sr}_{2} \mathrm{CoSi}_{2} \mathrm{O}_{7}$ and $\mathrm{Ba}_{2} \mathrm{CoGe}_{2} \mathrm{O}_{7}$, and the magnetic field dependence of $\mathbf{M}$ and $\mathbf{P}$ is also similar in the two compounds $[16,19]$, we use the same assignment of spin waves as for $\mathrm{Ba}_{2} \mathrm{CoGe}_{2} \mathrm{O}_{7}$ [20]. The $18-\mathrm{cm}^{-1}$ mode is the Goldstone mode of the easy-plane antiferromagnet gapped by the in-plane magnetic field whereas the latter two resonances correspond to the spin-stretching modes. When the field is parallel or antiparallel to the light propagation direction, the spectra are markedly different. The absorption difference is due to the MChD $[7,10]$.

As the temperature increases, the spin-stretching modes at 28 and $32 \mathrm{~cm}^{-1}$ merge and eventually disappear above $30 \mathrm{~K}$. However, the lowest-energy mode, the Goldstone mode
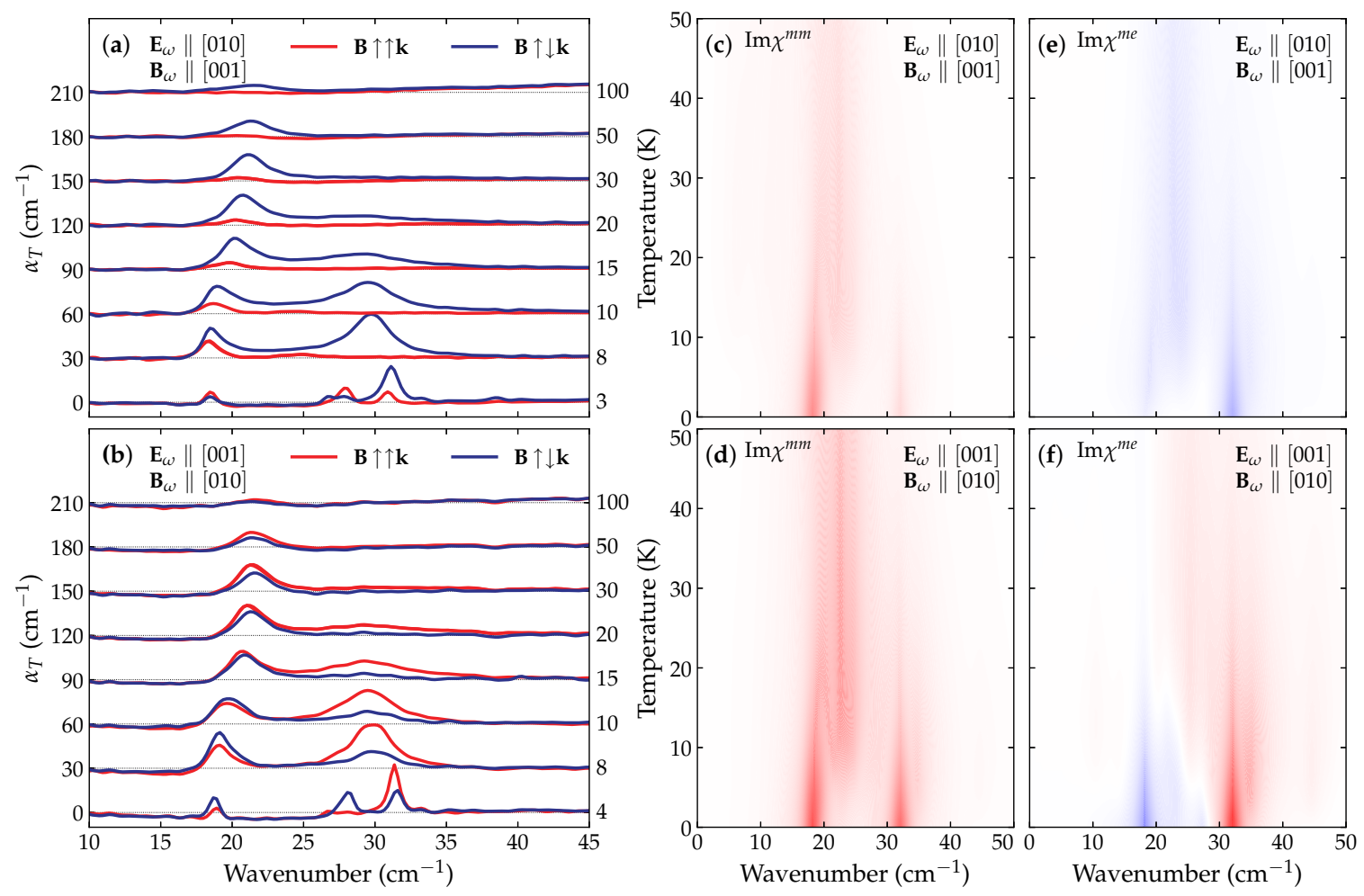

FIG. 2. Temperature dependence of measured $\mathrm{THz}$ absorption spectra (a), (b) and calculated susceptibilities (c) $-(\mathrm{f})$ of $\mathrm{Sr}_{2} \mathrm{CoSi}_{2} \mathrm{O}_{7}$ in $14 \mathrm{~T}$. The THz absorption spectra for magnetic field $\mathbf{B} \uparrow \uparrow \mathbf{k} \|[100]$ (red) and $\mathbf{B} \uparrow \downarrow \mathbf{k}$ (blue) are measured using linearly polarized radiation where in (a) $\mathbf{E}_{\omega} \|$ [010] and $\mathbf{B}_{\omega} \|$ [001], and in (b) the polarization is rotated by $\pi / 2$, so that $\mathbf{E}_{\omega} \|$ [001] and $\mathbf{B}_{\omega} \|$ [010]. The spectra measured at each temperature are shifted by a constant baseline. (c) and (e) are the magnetic susceptibility $\operatorname{Im} \chi^{m m}(\omega)$ and the ME susceptibility $\operatorname{Im} \chi^{m e}(\omega)$ for the polarizations $\mathbf{E}_{\omega} \|[010]$ and $\mathbf{B}_{\omega} \|$ [001]. (d) and (f) $\operatorname{are~} \operatorname{Im} \chi^{m m}(\omega)$ and $\operatorname{Im} \chi^{m e}(\omega)$ for the polarization $\mathbf{E}_{\omega} \|[001]$ and $\mathbf{B}_{\omega} \|[010]$. Red (positive) and blue (negative) colors indicate the sign of the susceptibility. The saturation of the color corresponds to the magnitude of the corresponding susceptibility matrix elements, $\operatorname{Im} \chi^{m m}(\omega)$ [Eq. (11)] and $\operatorname{Im} \chi^{m e}(\omega)$ [Eq. (12)]. The susceptibilities were calculated by the exact diagonalization of a four-site cluster. 

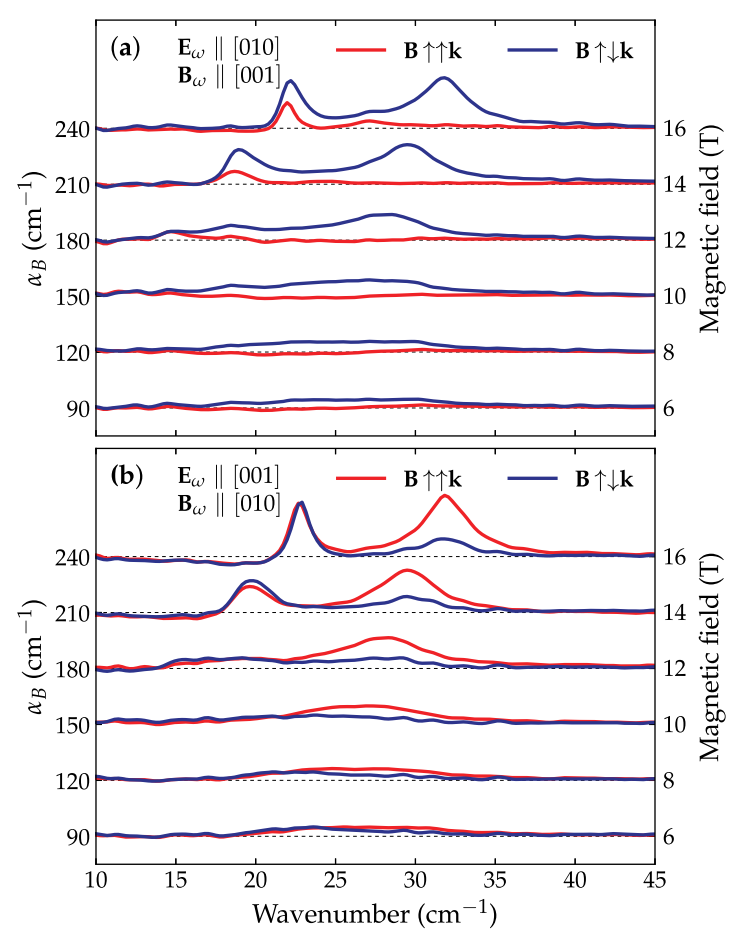

FIG. 3. Magnetic field dependence of absorption spectra of $\mathrm{Sr}_{2} \mathrm{CoSi}_{2} \mathrm{O}_{7}$ in the paramagnetic state at $10 \mathrm{~K}, \mathbf{B} \uparrow \uparrow \mathbf{k} \|[100]$ (red) and $\mathbf{B} \uparrow \downarrow \mathbf{k}$ (blue). The spectra are shifted in proportion to the absolute value of the magnetic field.

in the ordered phase, is visible even at $100 \mathrm{~K}$. The $\mathrm{MChD}$ has pronounced polarization dependence in the paramagnetic phase. A strong MChD is observed for all resonances in polarization $\mathbf{E}_{\omega} \|$ [010]: the absorption coefficient is nearly zero for positive fields whereas finite absorption is detected for negative fields. In the orthogonal polarization $\mathbf{E}_{\omega} \|$ [001], the lowest-energy resonance has weak $\mathrm{MChD}$ and changes sign between 10 and $15 \mathrm{~K}$.

In $14 \mathrm{~T}$ the $\mathrm{MChD}$ is the strongest at $10 \mathrm{~K}$, close to the Néel temperature but already in the paramagnetic phase [see Figs. 2(a) and 2(b)]. The magnetic field dependence of the absorption spectra at $10 \mathrm{~K}$ is shown in Figs. 3(a) and 3(b). The Goldstone-type mode suddenly appears above 12 $\mathrm{T}$ and it only shows $\mathrm{MChD}$ for $\mathbf{E}_{\omega} \|$ [010]. The remnant spin-stretching mode arises from the broad absorption feature in low magnetic field and has a strong MChD with opposite signs in the two polarizations.

The magnetic field dependence of the absorption spectra at $30 \mathrm{~K}$ is shown in Figs. 4(a) and 4(b). The average intensity of the single resonance line $\left(\alpha^{+}+\alpha^{-}\right) / 2$ observed at this temperature is nearly the same for both polarizations and it grows gradually as the field is increased. For polarization $\mathbf{E}_{\omega} \|[010]$ a strong $\mathrm{MChD}$ is observed while only a small absorption difference is detected in $\mathbf{E}_{\omega} \|$ [001].

\section{SPIN HAMILTONIAN AND MAGNETOELECTRIC COUPLING}

We consider the following spin Hamiltonian for $S=$ $\frac{3}{2} \mathrm{Co}^{2+}$ spins coupled within a single layer (Fig. 1) of

$$
\begin{aligned}
\mathrm{Sr}_{2} \mathrm{CoSi}_{2} \mathrm{O}_{7}: & \\
\mathcal{H}= & J \sum_{(i, j)}\left(S_{i}^{x} S_{j}^{x}+S_{i}^{y} S_{j}^{y}\right)+J_{z} \sum_{(i, j)} S_{i}^{z} S_{j}^{z}+\Lambda \sum_{i}\left(S_{i}^{z}\right)^{2} \\
& +D_{z} \sum_{(i, j)}\left(S_{i}^{x} S_{j}^{y}-S_{i}^{y} S_{j}^{x}\right)-g \mu_{\mathrm{B}} \mathbf{B} \sum_{i} \mathbf{S}_{i}
\end{aligned}
$$

where $J$ and $J_{z}$ are the anisotropic exchange parameters, $\Lambda$ is the onsite anisotropy parameter, $D_{z}$ is the $z$ component of the Dzyaloshinskii-Moriya vector, and the last term is the Zeeman interaction. $(i, j)$ denotes pairs of nearest-neighbor sites with $i \in \mathrm{A}$ and $j \in \mathrm{B}$ (Fig. 1). The spin-nematic interactions responsible for the weak in-plane anisotropy are neglected $[21,22]$.

From the symmetry point of view, the absence of inversion at the Co site allows the ME coupling. A suitable microscopic mechanism is provided by the spin-dependent $p-d$ hybridization [23]. In the case of a tetrahedrally coordinated magnetic moment (the $\mathrm{Co}^{2+}$ ion in our case), the electric polarization is quadratic in spin components given by $[19,24,25]$

$$
\mathbf{P} \propto \sum_{o=1}^{4}\left(\mathbf{S} \cdot \mathbf{e}_{o}\right)^{2} \mathbf{e}_{o},
$$

where $\mathbf{e}_{o}$ is the unit vector pointing from the center of the (distorted) tetrahedron toward the four $o=1, \ldots, 4$ ligands (the oxygen ions) at the vertices of the tetrahedron.

Since the $\mathrm{Sr}_{2} \mathrm{CoSi}_{2} \mathrm{O}_{7}$ is composed of alternating tetrahedra, the polarization components are

$$
\begin{aligned}
& P_{j}^{x} \propto-\cos 2 \kappa_{j}\left(S_{j}^{x} S_{j}^{z}+S_{j}^{z} S_{j}^{x}\right)-\sin 2 \kappa_{j}\left(S_{j}^{y} S_{j}^{z}+S_{j}^{z} S_{j}^{y}\right), \\
& P_{j}^{y} \propto \cos 2 \kappa_{j}\left(S_{j}^{y} S_{j}^{z}+S_{j}^{z} S_{j}^{y}\right)-\sin 2 \kappa_{j}\left(S_{j}^{x} S_{j}^{z}+S_{j}^{z} S_{j}^{x}\right), \\
& P_{j}^{z} \propto \cos 2 \kappa_{j}\left[\left(S_{j}^{y}\right)^{2}-\left(S_{j}^{x}\right)^{2}\right]-\sin 2 \kappa_{j}\left(S_{j}^{x} S_{j}^{y}+S_{j}^{y} S_{j}^{x}\right),
\end{aligned}
$$

where $j$ belongs to either sublattice A, with a tilt angle $\kappa_{j}=$ $\kappa$, or to sublattice B, where $\kappa_{j}=-\kappa$ [24].

The oscillating magnetic field of the light interacts with the total magnetization

$$
\mathbf{M}=\mathbf{M}_{\mathrm{A}}+\mathbf{M}_{\mathrm{B}},
$$

while the electric field interacts with the total polarization

$$
\mathbf{P}=\mathbf{P}_{\mathrm{A}}+\mathbf{P}_{\mathrm{B}},
$$

where the sublattice $(l=\mathrm{A}$ or $\mathrm{B})$ magnetization and polarization are

$$
\mathbf{M}_{l}=g \mu_{\mathrm{B}} \sum_{j \in l} \mathbf{S}_{j} \quad \text { and } \quad \mathbf{P}_{l}=\sum_{j \in l} \mathbf{P}_{j} .
$$

We define the magnetic susceptibility as

$$
\chi_{\mu \mu}^{m m}(\omega)=\sum_{i, f} \frac{\left|\left\langle f\left|M^{\mu}\right| i\right\rangle\right|^{2}}{\hbar \omega-E_{f}+E_{i}+i \delta} \frac{e^{-\beta E_{f}}-e^{-\beta E_{i}}}{Z},
$$

and the ME susceptibility as

$$
\chi_{\mu \nu}^{m e}(\omega)=\sum_{i, f} \frac{\left\langle i\left|M^{\mu}\right| f\right\rangle\left\langle f\left|P^{\nu}\right| i\right\rangle}{\hbar \omega-E_{f}+E_{i}+i \delta} \frac{e^{-\beta E_{f}}-e^{-\beta E_{i}}}{Z},
$$

where $Z=\sum_{i} e^{-\beta E_{i}}$ is the partition sum, $\beta=1 / k_{\mathrm{B}} T$ is the inverse temperature, the $\delta$ parameter gives a finite broadening 

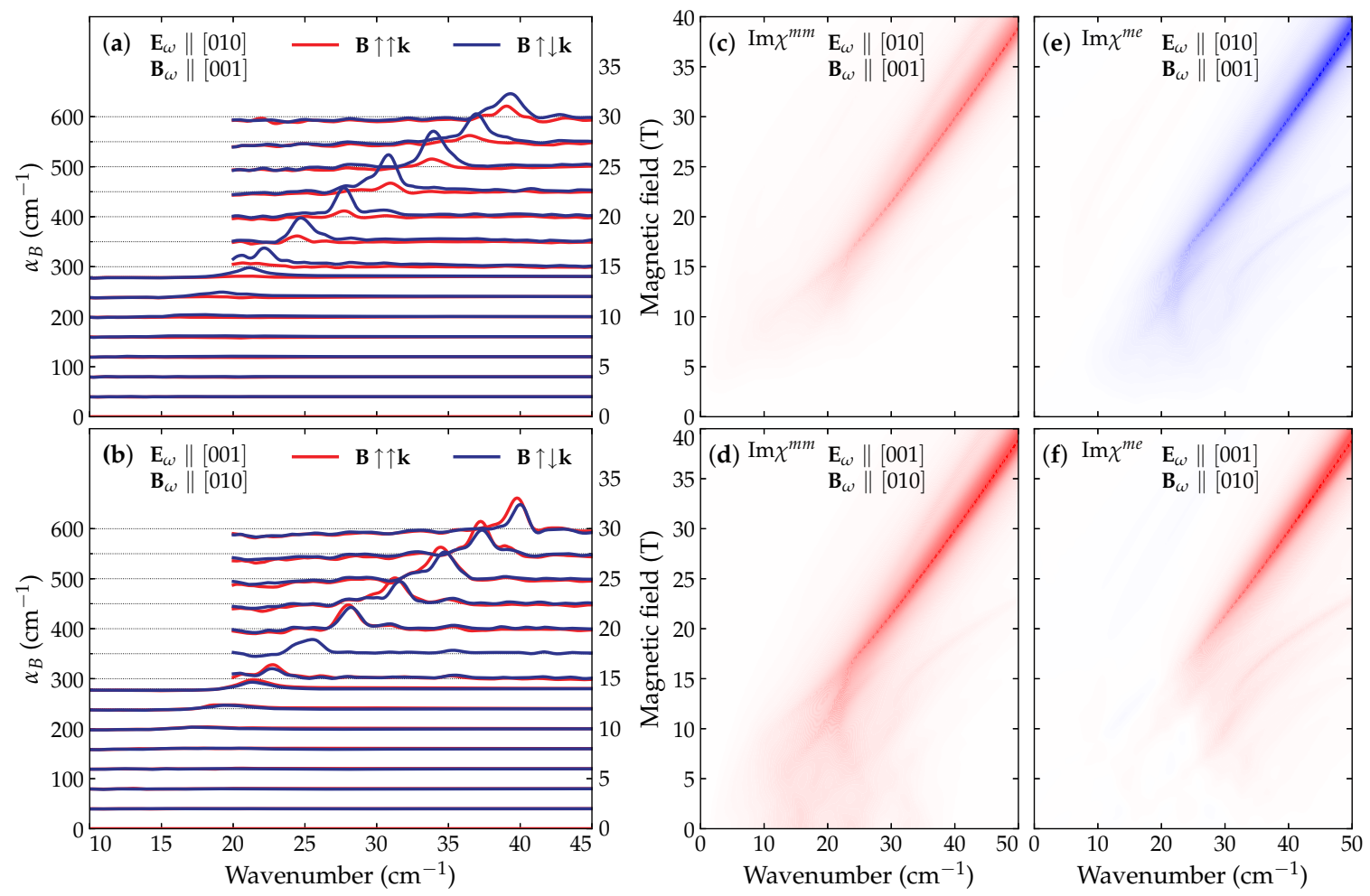

FIG. 4. Magnetic field dependence of measured $\mathrm{THz}$ absorption spectra (a), (b) and calculated susceptibilities (c)-(f) of $\mathrm{Sr}_{2} \mathrm{CoSi}_{2} \mathrm{O}_{7}$ at $30 \mathrm{~K}$. The THz absorption spectra for magnetic field $\mathbf{B} \uparrow \uparrow \mathbf{k} \|[100]$ (red) and $\mathbf{B} \uparrow \downarrow \mathbf{k}$ (blue) are shown for two polarizations of the THz light. The spectra are shifted in proportion to the absolute value of the magnetic field. (c) and (e) are the magnetic susceptibility $\operatorname{Im} \chi^{m m}(\omega)$ and the ME susceptibility $\operatorname{Im} \chi^{m e}(\omega)$ for the polarizations $\mathbf{E}_{\omega} \|[010]$ and $\mathbf{B}_{\omega} \|[001]$. (d) and (f) are $\operatorname{Im} \chi^{m m}(\omega)$ and $\operatorname{Im} \chi^{m e}(\omega)$ for the polarizations $\mathbf{E}_{\omega} \|$ [001] and $\mathbf{B}_{\omega} \|$ [010]. Red (positive) and blue (negative) colors indicate the sign of susceptibility. The saturation of the color corresponds to the magnitude of the corresponding susceptibility matrix elements $\operatorname{Im} \chi^{m m}(\omega)$ [Eq. (11)] and $\operatorname{Im} \chi^{m e}(\omega)$ [Eq. (12)]. The susceptibilities were calculated by the exact diagonalization of a four-site cluster.

to the absorption peaks, and $E_{i}$ and $E_{f}$ are the energies of the initial and final spin states, respectively.

The experimentally measured NDD is related to the imaginary and time-reversal odd part of ME susceptibility [13,26] as

$$
\alpha^{+}(\omega)-\alpha^{-}(\omega)=\frac{4 \omega}{c_{0}} \operatorname{Im} \chi_{\mu \nu}^{m e}(\omega),
$$

where $c_{0}$ is the speed of light in vacuum and frequency is in units of $\mathrm{rad} / \mathrm{s}$.

For a given transition $|i\rangle \rightarrow|f\rangle$ the imaginary (dissipative) parts of the magnetic and magnetoelectric susceptibilities are [13]

$$
\begin{gathered}
\operatorname{Im} \chi_{\mu \mu}^{m m}(\omega) \propto\left|\left\langle i\left|M^{\mu}\right| f\right\rangle\right|^{2} \delta\left(\omega-\omega_{i f}\right), \\
\operatorname{Im} \chi_{\mu \nu}^{m e}(\omega) \propto \operatorname{Re}\left\{\left\langle i\left|M^{\mu}\right| f\right\rangle\left\langle f\left|P^{v}\right| i\right\rangle\right\} \delta\left(\omega-\omega_{i f}\right),
\end{gathered}
$$

where $\omega_{i f}=\left(E_{f}-E_{i}\right) / \hbar$ is the transition frequency and $\omega$ is the frequency of a photon. Only the real part of the matrix element products is time-reversal odd in Eq. (12).

\section{EXACT DIAGONALIZATION}

To get a first insight into the nature of excitations, we performed an exact diagonalization study of a small cluster containing four $\mathrm{Co}^{2+}$ ions, i.e., two unit cells, at finite tem- peratures [27]. We note that exact diagonalization was also used to study the ME excitations of $\mathrm{Ba}_{2} \mathrm{CoGe}_{2} \mathrm{O}_{7}$ at zero temperature in Ref. [24].

Since the magnetization curves closely follow those of $\mathrm{Ba}_{2} \mathrm{CoGe}_{2} \mathrm{O}_{7}[16,19]$, we assume the same set of parameters describes both $\mathrm{Ba}_{2} \mathrm{CoGe}_{2} \mathrm{O}_{7}$ and $\mathrm{Sr}_{2} \mathrm{CoSi}_{2} \mathrm{O}_{7}$. We use the parameters obtained from absorption spectra of $\mathrm{Ba}_{2} \mathrm{CoGe}_{2} \mathrm{O}_{7}$ in Ref. [20], i.e., $\Lambda=13.4 \mathrm{~K}, J=2.3 \mathrm{~K}, J_{z z}=1.8 \mathrm{~K}, D_{z}=$ $-0.1 \mathrm{~K}, \kappa=22.4^{\circ}$, and $g=2.3$. This set of parameters provided a remarkable good agreement with the experimentally measured absorptions. The results of these calculations are presented in Figs. 2(c)-2(f) and Figs. 4(c)-4(f).

The exact diagonalization results show, in accordance with the experiment, that a single absorption line is present in high magnetic fields at high temperatures and a second resonance appears as the temperature is lowered below $20 \mathrm{~K}$. Calculation predicts a finite ME effect responsible for the observed NDD. Moreover, the sign change of NDD observed for $\mathbf{E}_{\omega} \|[001]$ between 10 and $15 \mathrm{~K}$ is reproduced by the numerical calculations [Figs. 2(b) and 2(f)].

\section{SINGLE-ION PROBLEM}

In order to get a deeper understanding of the $\mathrm{THz}$ absorption spectra and NDD in the paramagnetic phase of $\mathrm{Sr}_{2} \mathrm{CoSi}_{2} \mathrm{O}_{7}$, we consider a model of a single $\mathrm{Co}^{2+}$ spin in 


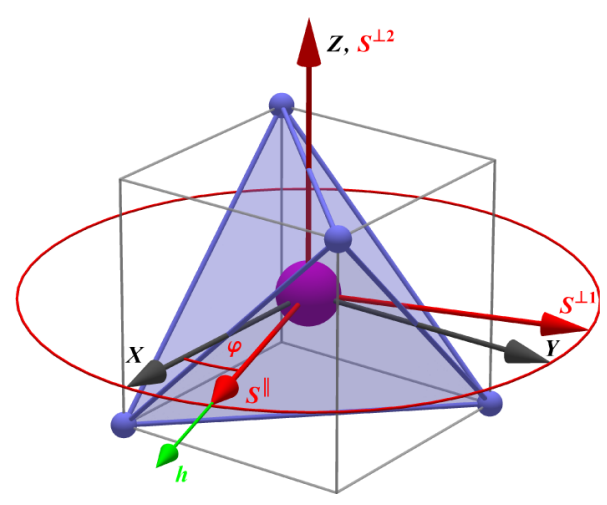

FIG. 5. The tetrahedron-fixed coordinate system $\{X, Y, Z\}$ and the rotated magnetic-field-fixed coordinate system $\{\|, \perp 1, \perp 2\}$. Purple ball is the $\mathrm{Co}^{2+}$ and the blue tetrahedron is the cage with the oxygen ions at the vertices. The quantization axis (spin component $S^{\|}$) is parallel to the direction of the static external magnetic field $h$ (shown in green), while one perpendicular axis (the spin component $S^{\perp 2}$ ) is parallel to $Z$.

the center of a tetrahedron. This unit is the building block of the crystal lattice of $\mathrm{Sr}_{2} \mathrm{CoSi}_{2} \mathrm{O}_{7}$, and, at the same time, its symmetry is representative of the field-induced reduction of the point symmetry in the real material. More precisely, in the next two sections we will show that the magnetic space group at the $\Gamma$ point is isomorphic to the magnetic point group of the tetrahedron. Furthermore, such a simple single-ion model describes the essence of the experiments in the disordered paramagnetic state where the strong thermal fluctuations smear out the coupling to the neighboring spins.

\section{A. Hamiltonian and the symmetries}

\section{Spin Hamiltonian and the electric polarization}

We will consider magnetic field in the $X Y$ plane of a single tetrahedron with a coordinate system defined in Fig. 5 . It is convenient to use the coordinate system where the $\|$ axis points in the direction of the field $h=g \mu_{\mathrm{B}} B$, the $\perp 2$ axis is parallel to the $Z$ direction, and the $\perp 1$ direction is chosen so that the three axes form an orthogonal right-handed system. The spin components in the coordinate system of the tetrahedron are related to the spin components in the fieldfixed system as

$$
\begin{aligned}
& S^{X}=\cos \varphi S^{\|}-\sin \varphi S^{\perp 1}, \\
& S^{Y}=\sin \varphi S^{\|}+\cos \varphi S^{\perp 1}, \\
& S^{Z}=S^{\perp 2} .
\end{aligned}
$$

Following Eq. (2) the Hamiltonian for a single spin in the field-fixed coordinates is

$$
\mathcal{H}=\Lambda\left(S^{\perp 2}\right)^{2}-h S^{\|} .
$$

The quantization axis is chosen along the magnetic field with eigenvalues and eigenvectors of $S^{\|}$being $\{+3 / 2,+1 / 2,-1 / 2,-3 / 2\}$ and $\{\Uparrow, \uparrow, \downarrow, \downarrow\}$, respectively.

The magnetic field and the anisotropy lower the $\mathrm{O}(3)$ spatial symmetry to the group generated by inversion and a twofold rotation $C_{2}^{\|}$about the magnetic field. Time-reversal symmetry alone is broken, but time reversal followed by a
TABLE I. Character table of the double group corresponding to the group $C_{2}$, including the transformation properties of the operators and states. The group element $\overline{\mathbf{1}}$ is the $2 \pi$ rotation with the property $\overline{\mathbf{1}}^{2}=\mathbf{1}$.

\begin{tabular}{lrrrrc}
\hline \hline Irrep & $\mathbf{1}$ & $C_{2}^{\|}$ & \multicolumn{1}{c}{$\overline{\mathbf{1}}$} & $\bar{C}_{2}^{\|}$ & Operators \\
\hline $\mathrm{A}$ & 1 & 1 & 1 & 1 & $S^{\|}, P^{\|}$ \\
$\mathrm{B}$ & 1 & -1 & 1 & -1 & $S^{\perp 1}, S^{\perp 2}, P^{\perp 1}, P^{\perp 2}$ \\
$\overline{\mathrm{E}}_{1}$ & 1 & $i$ & -1 & $-i$ & $|\downarrow\rangle,|\uparrow\rangle,\langle\Uparrow|,\langle\downarrow|$ \\
$\overline{\mathrm{E}}_{2}$ & 1 & $-i$ & -1 & $i$ & $|\Uparrow\rangle,|\downarrow\rangle,\langle\Downarrow|,\langle\uparrow|$ \\
\hline \hline
\end{tabular}

rotation perpendicular to the field, denoted by $\Theta C_{2}^{\perp 2}$, remains a symmetry element.

From Eq. (3) we get for the electric polarization

$$
\begin{aligned}
& P^{X}=\eta_{X Y}\left(S^{Z} S^{Y}+S^{Y} S^{Z}\right), \\
& P^{Y}=\eta_{X Y}\left(S^{Z} S^{X}+S^{X} S^{Z}\right), \\
& P^{Z}=\eta_{Z}\left(S^{X} S^{Y}+S^{Y} S^{X}\right),
\end{aligned}
$$

where for the regular tetrahedron $\eta_{X Y}=\eta_{Z}$. The relation to the components in the field-fixed coordinates is

$$
\begin{aligned}
& P^{X}=\cos \varphi P^{\|}-\sin \varphi P^{\perp 1}, \\
& P^{Y}=\sin \varphi P^{\|}+\cos \varphi P^{\perp 1}, \\
& P^{Z}=P^{\perp 2} .
\end{aligned}
$$

\section{Solution of the Hamiltonian}

The matrix representation of the Hamiltonian (14) in the spin basis $\{\Uparrow, \uparrow, \downarrow, \Downarrow\}$ is

$$
\hat{\mathcal{H}}=\left(\begin{array}{cccc}
\frac{3}{4} \Lambda-\frac{3}{2} h & 0 & -\frac{\sqrt{3}}{2} \Lambda & 0 \\
0 & \frac{7}{4} \Lambda-\frac{1}{2} h & 0 & -\frac{\sqrt{3}}{2} \Lambda \\
-\frac{\sqrt{3}}{2} \Lambda & 0 & \frac{7}{4} \Lambda+\frac{1}{2} h & 0 \\
0 & -\frac{\sqrt{3}}{2} \Lambda & 0 & \frac{3}{4} \Lambda+\frac{3}{2} h
\end{array}\right) .
$$

The eigenvalues $\pm i$ of the rotation operator $\hat{C}_{2}^{\|}$,

$$
\hat{C}_{2}^{\|}=e^{i \pi \hat{S}^{\|}}=\left(\begin{array}{cccc}
-i & 0 & 0 & 0 \\
0 & i & 0 & 0 \\
0 & 0 & -i & 0 \\
0 & 0 & 0 & i
\end{array}\right)
$$

are good quantum numbers as the operator commutes with the Hamiltonian $\left[\hat{\mathcal{H}}, \hat{C}_{2}^{\|}\right]=0$. Therefore, only the states with the same eigenvalue of $\hat{C}_{2}^{\|}$are mixed. We use $\pm i$ to label the eigenstates $\left|\psi_{j}^{( \pm i)}\right\rangle$ and corresponding energies $\varepsilon_{j}^{( \pm i)}$. For the detailed form of the energies and eigenvectors, we refer the reader to Appendix B 1.

\section{Transition matrix elements}

Based on the transformation properties under the rotation $C_{2}^{\|}$, as summarized in Table $\mathrm{I}$, we can construct selection rules for the matrix elements of the spin and polarization components. A matrix element for an operator $\mathcal{O}$ between states $\left|\psi_{\alpha}\right\rangle$ and $\left|\psi_{\beta}\right\rangle$ can only be nonvanishing if it transforms according to the totally symmetric $A$ irrep of the group 


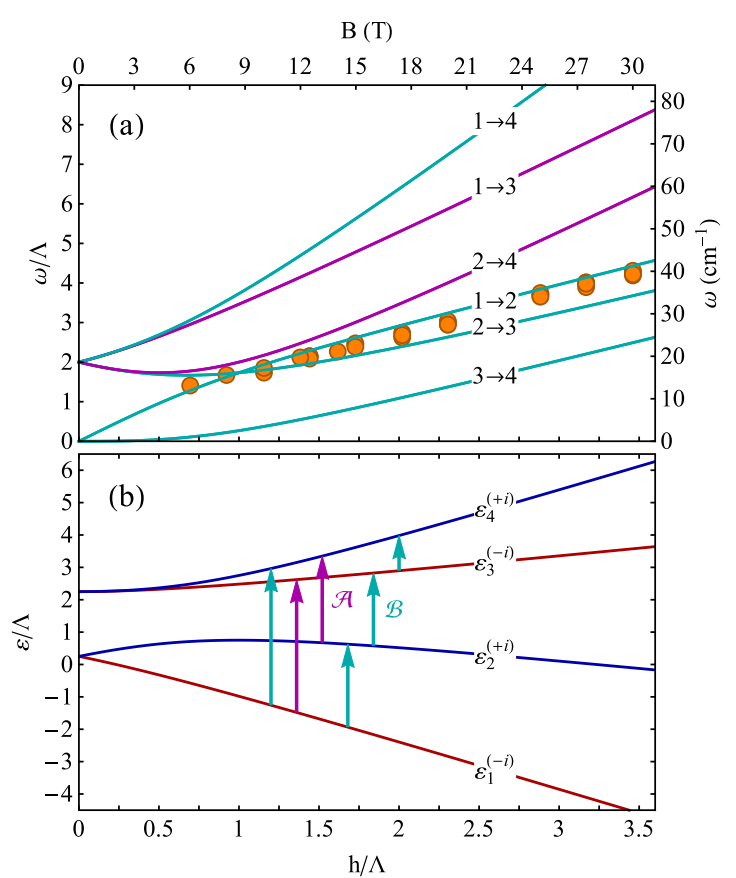

FIG. 6. (a) Transition energies $\omega$ and (b) energy levels $\varepsilon$ of the single-ion model with easy-plane anisotropy $\Lambda$ in a magnetic field $h=g \mu_{\mathrm{B}} B$ within the easy plane as a function of $h / \Lambda$, with $\Lambda=$ 13.4 $\mathrm{K}$ and $g=2.3$ from Ref. [20]. States 1 and 3 (red curves) are multiplied by $-i$ after a $\pi$ rotation about the field $\left(C_{2}^{\|}\right)$, while states 2 and 4 (blue) get an $i$. Magenta arrows represent transitions between the states of the same symmetry, induced by operator $\mathcal{A}$, even under the $C_{2}^{\|}$, such as $S^{\|}, P^{\|}$(see Table I). Cyan arrows connect states with different symmetries induced by operator $\mathcal{B}$, odd under $C_{2}^{\|}$, such as the perpendicular components of spin and polarization operator. The color of the curves corresponds to the color of the arrows in (b). The filled circles show the experimental results.

$A \subseteq \Gamma^{\beta} \otimes \Gamma(\mathcal{O}) \otimes \Gamma^{\alpha}$. In order to describe the transformation properties of the states of a half-integer spin, we need to consider the double group corresponding to the group $C_{2}$. If an operator $\mathcal{A}$ transforms as $\mathrm{A}$ and an operator $\mathcal{B}$ as $\mathrm{B}$, and the spin states as $\overline{\mathrm{E}}_{1}$ and $\overline{\mathrm{E}}_{2}$ of the double group of $C_{2}$ (Table I), the nonvanishing matrix elements are

$$
\left\langle\psi_{\alpha}^{( \pm i)}|\mathcal{A}| \psi_{\beta}^{( \pm i)}\right\rangle \quad \text { and } \quad\left\langle\psi_{\alpha}^{(\mp i)}|\mathcal{B}| \psi_{\beta}^{( \pm i)}\right\rangle .
$$

Allowed transitions between states of the same symmetry are of type $\mathcal{A}$ and allowed transitions between states of different symmetry are of type $\mathcal{B}$. The corresponding transition energies are shown in Fig. 6(a) and the energy levels involved in Fig. 6(b).

In what follows, we take into account the effects of antiunitary symmetries containing the time-reversal operation $\Theta$ on the matrix elements. We show in the Appendix A that any linear operator $\hat{\mathcal{O}}$, if the symmetry operation $\Theta C_{2}^{\perp 2}$ is present (as in the case of a magnetic field applied in the $X Y$ plane), must satisfy $\hat{\mathcal{O}}= \pm \hat{\mathcal{O}}^{*}$. Thus, the matrix elements of any linear operator even (odd) under the symmetry operation $\Theta C_{2}^{\perp 2}$ are real (imaginary).

In conclusion, the unitary symmetries of the single-ion model determine the selection rules, i.e., the nonvanishing
TABLE II. Matrix elements of the spin and polarization operators, first in small field $h$, then for small anisotropy $\Lambda$, in the single-ion model. The first nonvanishing order in either $h$ or $\Lambda$ is shown. $c$ stands for a real constant.

\begin{tabular}{|c|c|c|c|c|c|c|c|}
\hline Transition & $S^{\|}$ & $S^{\perp 1}$ & $S^{\perp 2}$ & $P_{\text {chiral }}^{\|}, P_{\text {polar }}^{\perp 1}$ & $P_{\text {chiral }}^{\perp 1}, P_{\text {polar }}^{\|}$ & $P_{\text {chiral }}^{\perp 2}$ & $P_{\text {polar }}^{\perp 2}$ \\
\hline $1 \rightarrow 2$ & & $c, c$ & $i c, i c$ & & $i h, i c$ & $h, c$ & \\
\hline $2 \rightarrow 3$ & & $c, c$ & $i h, i c$ & & $i c, i \Lambda$ & $c, \Lambda$ & \\
\hline $3 \rightarrow 4$ & & $h^{2}, c$ & $i c, i c$ & & $i h, i c$ & $h, c$ & \\
\hline $1 \rightarrow 3$ & $c, \Lambda$ & & & $i c, i c$ & & & $c, c$ \\
\hline $2 \rightarrow 4$ & $c, \Lambda$ & & & $i c, i c$ & & & $c, c$ \\
\hline $1 \rightarrow 4$ & & $c, \Lambda^{2}$ & $i h, i \Lambda^{2}$ & & $i c, i \Lambda$ & $c, \Lambda$ & \\
\hline
\end{tabular}

matrix elements, whereas the antiunitary symmetry constrains the matrix element to a real or to an imaginary value.

\section{B. Directional dichroism in the single-ion model}

Imaginary part of the ME susceptibility $\operatorname{Im} \chi_{\mu \nu}^{m e}(\omega)$ causes $\operatorname{NDD}[6,7,28]$ [Eq. (10)]. For a given transition $|i\rangle \rightarrow|f\rangle$ the dissipative and time-reversal odd part of the ME susceptibility is proportional to the real part of the matrix element product $\operatorname{Re}\left\{\left\langle f\left|M^{\mu}\right| i\right\rangle\left\langle f\left|P^{v}\right| i\right\rangle\right\}$ [Eq. (12)]. Thus, NDD is nonzero if both matrix elements are nonzero for the same pair of states $|i\rangle$ and $|f\rangle$. This is allowed by symmetry if $M^{\mu}$ and $P^{v}$ transform according to the same irreducible representation of the group of unitary symmetries.

Furthermore, the product of the $M^{\mu}$ and $P^{v}$ matrix elements has a finite real part only if both matrix elements are real or both are imaginary. Thus, both operators must be even ( $\mathcal{A}$ type) or both odd ( $\mathcal{B}$ type) under antiunitary symmetry operation, as was shown in the previous section. The summary of all spin and polarization operator amplitudes for $S=\frac{3}{2}$ in the single-ion model is presented in Table II. They are either real, imaginary, or symmetry forbidden.

Below we examine the symmetry properties of the operators for different directions of the external field with respect to the cobalt-oxygen tetrahedron in the easy plane to find out the details of the existence of NDD.

\section{Magnetochiral dichroism (chiral case): $\varphi=0$}

When the external field is parallel to one of the twofold rotation axes $\varphi=0$ (see Fig. 5), the system has chiral symmetry $D_{2}\left(C_{2}\right)$ in Schoenflies notation [see Fig. 7(a)], and the polarization operators in the local coordinate system are

$$
\begin{aligned}
& P_{\text {chiral }}^{\|}=\frac{\eta_{X Y}}{2 i}\left[\left(S^{+}\right)^{2}-\left(S^{-}\right)^{2}\right], \\
& P_{\text {chiral }}^{\perp 1}=\frac{\eta_{X Y}}{2 i}\left[S^{\|}\left(S^{+}-S^{-}\right)+\left(S^{+}-S^{-}\right) S^{\|}\right], \\
& P_{\text {chiral }}^{\perp 2}=\frac{\eta_{Z}}{2}\left[S^{\|}\left(S^{+}+S^{-}\right)+\left(S^{+}+S^{-}\right) S^{\|}\right],
\end{aligned}
$$

where $S^{ \pm}=S^{\perp 1} \pm i S^{\perp 2}$. This is also the case (up to a sign) when $\varphi$ is an integer multiple of $\pi / 2$ due to the $S_{4}$ symmetry of the distorted oxygen tetrahedron. It is worth noting that the perpendicular components $P^{\perp 1}$ and $P^{\perp 2}$ change the $S^{\|}$ quantum number by \pm 1 , creating dipolar spin excitation, but 


\section{(a) chiral}

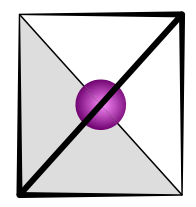

(b) achiral and apolar

(c) polar

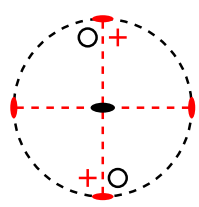

$\mathrm{D}_{2}\left(\mathrm{C}_{2}\right)$
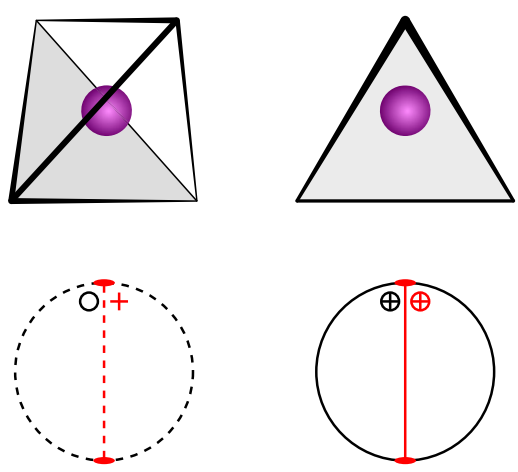

$\mathrm{D}_{1}\left(\mathrm{C}_{1}\right)$

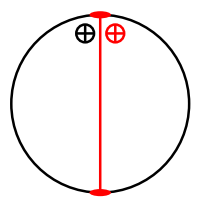

$\mathrm{C}_{2 \mathrm{v}}\left(\mathrm{C}_{1 \mathrm{~h}}\right)$

FIG. 7. The tetrahedron in the (a) chiral $(\varphi=0)$, (b) lowsymmetry, and (c) polar $(\varphi=\pi / 4)$ cases as seen from the direction of the magnetic field $\mathbf{B}$. The magenta sphere represents the magnetic ion at the center of tetrahedral cage. The stereograms represent the magnetic point group for each case, black refers to the unitary subgroup, and red the antiunitary part combined with the time reversal.

$P_{\text {chiral }}^{\|}$changes by \pm 2 , creating quadrupolar spin excitation [29].

None of the components of $\mathbf{P}$ transform according to the fully symmetric irreducible representation $A_{+}$(Table III). Therefore, the expectation value for the static polarization is zero in the ground state. Although the chiral case is apolar, the dynamic ME susceptibility is allowed. The operators $S^{\perp 2}$ and $P^{\perp 1}$ belong to the same irreducible representation $B_{-}$of $D_{2}\left(C_{2}\right)$, thus, the dynamic ME susceptibility is allowed. As the oscillating magnetization $S^{\perp 2}$ and polarization $P^{\perp 1}$ are perpendicular to each other and to the external magnetic field, we expect NDD in the Faraday geometry, when the light is propagating parallel to the field. This effect is nothing else

TABLE III. Character table for the magnetic point groups and symmetry-allowed operators for three orientations of the applied magnetic field in the easy plane (see Fig. 5), creating chiral $(\varphi=0)$, polar $(\varphi=\pi / 4)$, and low-symmetry cases.

\begin{tabular}{|c|c|c|c|c|c|c|}
\hline \multirow[b]{2}{*}{ Irrep. } & \multirow[b]{2}{*}{1} & \multicolumn{4}{|c|}{$\varphi=0:$ chiral case, the group is $D_{2}\left(C_{2}\right)$} & \multirow[b]{2}{*}{ NDD } \\
\hline & & $C_{2}^{\|}$ & $\Theta C_{2}^{\perp 2}$ & $\Theta C_{2}^{\perp 1}$ & Operator(s) & \\
\hline $\mathrm{A}_{+}$ & 1 & 1 & 1 & 1 & $S^{\|}$ & \\
\hline$A_{-}$ & 1 & 1 & -1 & -1 & $P^{\|}$ & \\
\hline $\mathrm{B}_{+}$ & 1 & -1 & 1 & -1 & $S^{\perp 1}, P^{\perp 2}$ & Faraday \\
\hline \multirow[t]{3}{*}{$\mathrm{B}_{-}$} & 1 & -1 & -1 & 1 & $S^{\perp 2}, P^{\perp 1}$ & Faraday \\
\hline & \multicolumn{5}{|c|}{$\varphi=\pi / 4:$ polar case, the group is $C_{2 v}\left(C_{1 h}\right)$} & \\
\hline & 1 & $\sigma^{\|}$ & $\Theta C_{2}^{\perp 2}$ & $\Theta \sigma^{\perp 1}$ & & \\
\hline$\overline{A_{+}}$ & 1 & 1 & 1 & 1 & $S^{\|}, P^{\perp 2}$ & Voigt \\
\hline$A_{-}$ & 1 & 1 & -1 & -1 & $P^{\perp 1}$ & \\
\hline $\mathrm{B}_{+}$ & 1 & -1 & 1 & -1 & $S^{\perp 1}$ & \\
\hline \multirow[t]{2}{*}{$\mathrm{B}_{-}$} & 1 & -1 & -1 & 1 & $S^{\perp 2}, P^{\|}$ & Voigt \\
\hline & \multicolumn{5}{|c|}{$\varphi \neq 0$ nor $\pi / 4:$ the group is $D_{1}\left(C_{1}\right)$} & \\
\hline$\overline{\Gamma_{+}}$ & 1 & & 1 & & $S^{\|}, S^{\perp 1}, P^{\perp 2}$ & Both \\
\hline$\Gamma_{-}$ & 1 & & -1 & & $S^{\perp 2}, P^{\|}, P^{\perp 1}$ & Both \\
\hline
\end{tabular}

but the MChD. The operators $S^{\perp 1}$ and $P^{\perp 2}$, corresponding to the perpendicular polarization of radiation as compared to $S^{\perp 2}$ and $P^{\perp 1}$, belong to the same irreducible representation $\mathrm{B}_{+}$and therefore the NDD will appear irrespective of polarizations of the incident light in Faraday geometry. The symmetry argument presented above is supported by the direct evaluation of the matrix elements (see Appendix B and Table II) using the eigenstates given in Eqs. (B2a)-(B2d).

From Table III we can also infer the selection rules with regard to the quantum numbers $\pm i$, the eigenvalues of the rotation operator $C_{2}^{\|}$. The $S^{\|}$and $P^{\|}$are invariant under $C_{2}^{\|}$, thus, they excite only type- $\mathcal{A}$ transitions between states that have the same quantum number [see Fig. 6(b)], i.e., between states 1 and 3 and between states 2 and 4 . However, there is no NDD for the $\mathcal{A}$-type transitions as $P^{\|}$and $S^{\|}$do not transform according to the same irrep.

The perpendicular components of $\mathbf{P}$ and $\mathbf{S}$ have matrix elements between states with different $C_{2}^{\|}$quantum numbers, which corresponds to type- $\mathcal{B}$ transitions, i.e., transitions $1 \rightarrow$ $2,1 \rightarrow 4,2 \rightarrow 3$, and $3 \rightarrow 4$ [see Fig. 6(b)]. We also see in Table III that $S^{\perp 2}$ and $P^{\perp 1}$ are both odd under $\Theta C_{2}^{\perp 2}$, with imaginary matrix elements, and their product is real in Eq. (12), providing a finite imaginary $\chi_{\perp 2, \perp 1}^{m e}(\omega)$ and a finite MChD. Similarly, the $S^{\perp 1}$ and $P^{\perp 2}$ are both even, with real matrix elements, providing a finite imaginary $\chi_{\perp 1, \perp 2}^{m e}(\omega)$. In any other configuration the $\operatorname{Im}\left\{\chi_{\mu \nu}^{m e}(\omega)\right\}=0$. Therefore, the NDD is present only in the Faraday configuration, $\mathbf{k} \| \mathbf{B}$.

\section{Toroidal dichroism (polar case): $\varphi=\frac{\pi}{4}$}

If the field direction is parallel to the upper edge of the tetrahedron, $\varphi=\frac{\pi}{4}$ in Fig. 5, and the polarization operators are

$$
\begin{aligned}
P_{\text {polar }}^{\|} & =\frac{\eta_{X Y}}{2 i}\left[S^{\|}\left(S^{+}-S^{-}\right)+\left(S^{+}-S^{-}\right) S^{\|}\right] \\
P_{\text {polar }}^{\perp 1} & =\frac{\eta_{X Y}}{2 i}\left[\left(S^{-}\right)^{2}-\left(S^{+}\right)^{2}\right] \\
P_{\text {polar }}^{\perp 2} & =\frac{\eta_{Z}}{4}\left[4\left(S^{\|}\right)^{2}-\left(S^{-}\right)^{2}-\left(S^{+}\right)^{2}-S^{-} S^{+}-S^{+} S^{-}\right] .
\end{aligned}
$$

Here, the perpendicular operators are changing the $S^{\|}$quantum number by 0 and \pm 2 , and the $P^{\|}$parallel operator by \pm 1 . We note that $P_{\text {polar }}^{\|}=P_{\text {chiral }}^{\perp 1}$ and $P_{\text {polar }}^{\perp 1}=-P_{\text {chiral }}^{\|}$, reflecting the spin-quadrupolar nature of the polarization operators.

As shown in Fig. 7(c), the symmetry group of the spin Hamiltonian is now $C_{2 v}\left(C_{1 h}\right)=2^{\prime} m^{\prime} m$ with elements

$$
C_{2 v}\left(C_{1 h}\right)=2^{\prime} m^{\prime} m=\left\{\mathbf{1}, \sigma^{\|}, \Theta C_{2}^{\perp 2}, \Theta \sigma^{\perp 1}\right\} .
$$

The character table and the transformations of physical quantities under this group are given in Table III.

If we compare to the chiral case, first, we observe that the $P^{\perp 2}$ transforms as identity, therefore electric polarization along $\perp 2$ axis is now allowed by the symmetry, hence we refer to this case as the polar case. Second, because of the $\sigma^{\|}$symmetry element the $S^{\perp 1}$ and $P^{\perp 2}$ do not belong to the same irreducible representation anymore, and similarly $S^{\perp 2}$ and $P^{\perp 1}$. As a consequence, the NDD in the Faraday geometry vanishes. Indeed, in Faraday geometry $\mathbf{k} \| \mathbf{B}$, the 
THz field transforms under $\sigma^{\|}$as $\mathbf{B}_{\omega} \rightarrow-\mathbf{B}_{\omega}, \mathbf{E}_{\omega} \rightarrow \mathbf{E}_{\omega}$, and $\mathbf{k} \rightarrow-\mathbf{k}$. The two directions of the radiation propagation are connected by the symmetry element and NDD is forbidden $[5,13]$.

Instead, the $S^{\perp 2}$ and $P^{\|}$transform according to the same irreducible representation $B_{-}$. As a consequence, a finite $\mathrm{ME}$ susceptibility $\chi_{\perp 2, \|}^{m e}$ will appear in the Voigt geometry when the $\mathbf{k}$ is parallel to the $\perp 1$ and $\mathbf{E}_{\omega} \| \mathbf{B}$. Similarly, the $S^{\|}$and $P^{\perp 2}$ both belong to $A_{+}$, providing a finite $\chi_{\|, \perp 2}^{m e}$. Since the operators belonging to the same irreducible representation in Table III have the same parity under $\Theta C_{2}^{\perp 2}$ transformation, their matrix elements are either both real (for $S^{\perp 2}$ and $P^{\|}$), or are both pure imaginary (for $S^{\|}$and $P^{\perp 2}$ ), allowing NDD in the Voigt configuration in both polarizations of $\mathrm{THz}$ radiation.

As the $\sigma^{\|}$and $C_{2}^{\|}$are given by the same matrix in Eq. (18) [30], we can repeat the arguments we used in the chiral case to determine the selection rules. The $S^{\|}$and $P^{\perp 2}$ operators have finite matrix elements between states with the same $\hat{C}_{2}^{\|} \equiv \hat{\sigma}^{\|}$ quantum number, i.e., the $\mathcal{A}$-type transitions $1 \rightarrow 3$ and $2 \rightarrow$ 4 are allowed. The $S^{\perp 2}$ and $P^{\|}$change the quantum number $\pm i \rightarrow \mp i$ and the $\mathcal{B}$-type transitions $1 \rightarrow 2,1 \rightarrow 4,2 \rightarrow 3$, and $3 \rightarrow 4$ are allowed.

\section{Low-symmetry case: $\varphi \neq 0$ nor $\frac{\pi}{4}$}

For arbitrary direction of the external magnetic field within the $X Y$ plane, the polarization operators can be written as a linear combination of the chiral and polar cases considered above:

$$
\mathbf{P}=\cos 2 \varphi \mathbf{P}_{\text {chiral }}+\sin 2 \varphi \mathbf{P}_{\text {polar }}
$$

Here, only the $\Theta C_{2}^{\perp 2}$ symmetry remains and the magnetic point group is reduced to the $D_{1}\left(C_{1}\right)=2^{\prime}$ [see Table III and Fig. 7(b)]. Since neither $C_{2}^{\|}$nor $\sigma^{\|}$is a symmetry element of the full problem, the $\mathbf{P}$ may have finite matrix elements between any of the $( \pm i)$ states, and the selection rules we established for the chiral $(\varphi=0)$ and the polar $(\varphi=\pi / 4)$ case are not valid. However, as the spin state still respects the quantum number set by the $C_{2}^{\|}$, the associated selection rules [Eq. (19)] hold for $S^{\|}, S^{\perp 1}$, and $S^{\perp 2}$. Furthermore, since the ME susceptibility is composed from a product of matrix elements of $\mathbf{S}$ and $\mathbf{P}$, it inherits the selection rules of the matrix element of $\mathbf{S}$. Putting all this together, the single-ion system shows NDD in the Faraday geometry for the $1 \rightarrow$ $2,2 \rightarrow 3,1 \rightarrow 4$, and $3 \rightarrow 4$ transitions with $\chi^{m e} \propto \cos 2 \varphi$ coming from the chiral part, and NDD in the Voigt geometry according to the selection rules set by the polar case with $\chi^{m e} \propto \sin 2 \varphi$.

\section{LATTICE PROBLEM}

This section describes the selection rules when the tetrahedra form a lattice. Furthermore, we give the analytical form of the transition energies by taking into account the exchange coupling between the spins in the lowest order in perturbation theory.

\section{A. Magnetochiral dichroism and selection rules in the lattice problem}

In the material the oxygen tetrahedra are rotated alternatingly, there is no direction of the external field which would show purely either the chiral or the polar case discussed in the section above. However, the situation is not hopeless: if the external field is along the [100] direction (as in the actual experiment), there is a $\left\{C_{2}^{\|} \mid\left[\frac{1}{2} 00\right]\right\}$ screw axis (shown in Fig. 1 as $2_{1}$ ) which is a symmetry operation. The screw axis performs a $\pi$ rotation about the [100] axis and a half-translation along the same axis to move the A tetrahedra into $\mathrm{B}$ and vice versa (see Fig. 1). The screw axis is a symmetry element of the lattice spin Hamiltonian symmetry group, so we can use its irreducible representations to label the eigenstates and the operators. In addition, the $\left\{\Theta C_{2}^{\perp 2} \mid[000]\right\}$ (the rotation by $\pi$ about the [001] axes through the center of a tetrahedron followed by a time-reversal operation) is a symmetry element irrespectively from the direction of the in-plane magnetic field. In fact, this nonsymmorphic magnetic point group is isomorphic to the $D_{2}\left(C_{2}\right)=22^{\prime} 2^{\prime}$ magnetic point group of a single tetrahedron in the chiral case:

$$
\begin{aligned}
& \left\{\mathbf{1},\left\{C_{2}^{\|} \mid\left[\frac{1}{2} 00\right]\right\},\left\{\Theta C_{2}^{\perp 2} \mid[000]\right\},\left\{\Theta C_{2}^{\perp 1} \mid\left[0 \frac{1}{2} 0\right]\right\}\right. \\
& \quad \cong\left\{\mathbf{1}, C_{2}^{\|}, \Theta C_{2}^{\perp 2}, \Theta C_{2}^{\perp 1}\right\} .
\end{aligned}
$$

Let us examine the selection rules based on what we learned for a single ion. We express the magnetization [Eq. (5)] and the polarization [Eq. (6)] in the magnetic-fieldfixed coordinate system and decompose into the irreducible representations of the unitary part of the point group, which consists of the identity and the $\left\{C_{2}^{\|} \mid\left[\frac{1}{2} 00\right]\right\}$ screw axis. The screw axis acts on the magnetization as

$$
\begin{aligned}
& \left(\begin{array}{c}
M_{\mathrm{A}}^{\|} \\
M_{\mathrm{A}}^{\perp 1} \\
M_{\mathrm{A}}^{\perp 2}
\end{array}\right) \rightarrow\left(\begin{array}{c}
M_{\mathrm{B}}^{\|} \\
-M_{\mathrm{B}}^{\perp 1} \\
-M_{\mathrm{B}}^{\perp 2}
\end{array}\right), \\
& \left(\begin{array}{c}
M_{\mathrm{B}}^{\|} \\
M_{\mathrm{B}}^{\perp 1} \\
M_{\mathrm{B}}^{\perp 2}
\end{array}\right) \rightarrow\left(\begin{array}{c}
M_{\mathrm{A}}^{\|} \\
-M_{\mathrm{A}}^{\perp 1} \\
-M_{\mathrm{A}}^{\perp 2}
\end{array}\right) .
\end{aligned}
$$

Similar considerations hold for the polarization operators.

Since the unitary part of the point group has two irreducible representations, the $\mathbf{M}$ operator can be decomposed into even $\left(\mathbf{M}_{\mathbf{0}}\right)$ and odd $\left(\mathbf{M}_{\pi}\right)$ parts as $\mathbf{M}=\mathbf{M}_{\mathbf{0}}+\mathbf{M}_{\pi}$.

\section{Selection rules for the even (0) components}

Using the transformation rules given by Eq. (25), the even part of $\mathbf{M}$ is

$$
\begin{aligned}
M_{\mathbf{0}}^{\|} & =M_{\mathrm{A}}^{\|}+M_{\mathrm{B}}^{\|}, \\
M_{\mathbf{0}}^{\perp} & =0,
\end{aligned}
$$

and for the polarizations we get

$$
\begin{aligned}
P_{\mathbf{0}}^{\|} & =\sin 2 \kappa \sum_{j}(-1)^{j} P_{j, \text { polar }}^{\|}-\cos 2 \kappa \sum_{j} P_{j, \text { chiral }}^{\|}, \\
P_{\mathbf{0}}^{\perp} & =0
\end{aligned}
$$


where $\perp=\perp 1, \perp 2$ and the index $j$ runs over all the sites, being an even integer on A and odd integer on the B sublattice; $P_{j \text {,chiral }}^{\|}$is defined by Eq. (20a) and $P_{j \text {,polar }}^{\|}$by Eq. (21a), with the corresponding spin operators at site $j$. The light does not interact with the system in the Faraday geometry in the even channel because $P_{\mathbf{0}}^{\perp}=M_{\mathbf{0}}^{\perp}=0$.

\section{Selection rules for the odd $(\pi)$ components}

When we antisymmetrize, we get the odd quantities $\mathbf{M}_{\boldsymbol{\pi}}$ and $\mathbf{P}_{\pi}$, and following the steps we used to obtain the even components above, the corresponding magnetization and polarization components are

$$
\begin{aligned}
& M_{\pi}^{\|}=0, \\
& M_{\pi}^{\perp}=M_{\mathrm{A}}^{\perp}+M_{\mathrm{B}}^{\perp}
\end{aligned}
$$

and

$$
\begin{aligned}
& P_{\pi}^{\|}=0, \\
& P_{\pi}^{\perp}=\sin 2 \kappa \sum_{j}(-1)^{j} P_{j, \text { polar }}^{\perp}-\cos 2 \kappa \sum_{j} P_{j, \text { chiral }}^{\perp} .
\end{aligned}
$$

The nonvanishing perpendicular components $\left\{M_{\pi}^{\perp 1}, P_{\pi}^{\perp 2}\right\}$ and $\left\{M_{\pi}^{\perp 2}, P_{\pi}^{\perp 1}\right\}$ lead to a finite ME susceptibility and NDD in the Faraday geometry for the odd channel.

We now examine the effect of the time reversal. The action of the $\left\{\Theta C_{2}^{\perp 2} \mid[000]\right\}$ is given by

$$
\begin{aligned}
& \left(\begin{array}{c}
M_{\mathrm{A}}^{\|} \\
M_{\mathrm{A}}^{\perp 1} \\
M_{\mathrm{A}}^{\perp 2}
\end{array}\right) \rightarrow\left(\begin{array}{c}
M_{\mathrm{A}}^{\|} \\
M_{\mathrm{A}}^{\perp 1} \\
-M_{\mathrm{A}}^{\perp 2}
\end{array}\right), \\
& \left(\begin{array}{c}
P_{\mathrm{A}}^{\|} \\
P_{\mathrm{A}}^{\perp 1} \\
P_{\mathrm{A}}^{\perp 2}
\end{array}\right) \rightarrow\left(\begin{array}{c}
-P_{\mathrm{A}}^{\|} \\
-P_{\mathrm{A}}^{\perp 1} \\
P_{\mathrm{A}}^{\perp 2}
\end{array}\right),
\end{aligned}
$$

and the same equations on the B sublattice. Just like in the single-ion problem, the $M_{\pi}^{\perp 1}$ and $P_{\pi}^{\perp 2}$ belong to the same irreducible representation, as well as the $M_{\pi}^{\perp 2}$ and $P_{\pi}^{\perp 1}$. The matrix elements are therefore real or imaginary, and the product of the magnetization and polarization matrix elements in the ME susceptibility is real.

Although the symmetry classification obtained above did not consider the DM interaction, it describes the selection rules obtained from the exact diagonalization. This is because the DM interaction is compatible with the $D_{2}\left(C_{2}\right)$ magnetic point group considered above.

\section{B. Perturbative effects of the exchange coupling}

So far, we have neglected the interactions between the Co spins. To assess how much the single-ion picture is altered, we take into account the effect of exchange couplings perturbatively: starting from the single-ion limit, $J=J_{z}=D_{z}=0$ in Eq. (2), we derive a tight-binding-like approximation for the exchange part. We denote the onsite and exchange parts as $\mathcal{H}^{0}$ and $\mathcal{H}^{\prime}$.
For the noninteracting case, the ground state of $\mathcal{H}^{0}$ is

$$
\left|\Psi_{1}^{0}\right\rangle=\prod_{j}\left|\psi_{1}^{(-i)}(j)\right\rangle,
$$

where $j$ runs over both the A and B sublattice sites. We define the local single-ion excitation at site $l$ as

$$
\left|\Psi_{2}^{0}(l)\right\rangle=\left|\psi_{2}^{(+i)}(l)\right\rangle \prod_{j \neq l}\left|\psi_{1}^{(-i)}(j)\right\rangle .
$$

The local wave functions $\left|\psi_{\alpha}^{( \pm i)}(l)\right\rangle$ are given by Eq. (B2). If the number of (all) sites is $N$, the noninteracting groundstate energy is $E_{1}^{0}=N \varepsilon_{1}$ and the excited states $\left|\Psi_{2}^{0}(l)\right\rangle$ have energies $E_{2}^{0}=(N-1) \varepsilon_{1}+\varepsilon_{2}$ and are $N$-fold degenerate.

In order to calculate the degeneracy lifting in the first order of the degenerate perturbation expansion, we need to diagonalize the perturbing matrix $\mathcal{H}^{\prime}$ on the subspace spanned by $\left|\Psi_{2}^{0}(l)\right\rangle$,

$$
\left\langle\Psi_{2}^{0}\left(l^{\prime}\right)\left|\mathcal{H}^{\prime}\right| \Psi_{2}^{0}(l)\right\rangle
$$

describing a local excited state which hops with equal amplitudes in different directions. Generally, the hopping problem is diagonalized in the momentum space for a translationally symmetric problem. In our case, the translational symmetry holds for the unit cell containing two lattice sites, with translation vectors $\mathbf{t}_{1}^{\prime}=(1,1,0)$ and $\mathbf{t}_{2}^{\prime}=(1,-1,0)$ in the $(x, y, z)$ coordinate system (see Fig. 1). However, the $\left\{C_{2}^{\|} \mid\left[\frac{1}{2} 00\right]\right\}$ screw axis and the $\mathbf{t}_{1}^{\prime}$ and/or $\mathbf{t}_{2}^{\prime}$ translation generate an Abelian group isomorphic to the group constructed from the $\mathbf{t}_{1}=(1,0,0)$ and $\mathbf{t}_{2}=(0,1,0)$, the translations of the lattice of the Co ions, if we neglect the alternating tetrahedra. The irreducible representations are all one dimensional, with

$$
\left\{C_{2}^{\|} \mid\left[\frac{1}{2} 00\right]\right\}\left|\Psi_{\mathbf{k}}\right\rangle=e^{i \mathbf{k} \cdot \mathbf{t}_{1}}\left|\Psi_{\mathbf{k}}\right\rangle,
$$

where $\mathbf{k}$ plays the role of the momentum. As a result, the $\mathbf{k}=$ $(0,0)=\mathbf{0}$ and $\mathbf{k}=(\pi, \pi)=\pi$ states (which, in fact, are both at the $\Gamma$ point of the Brillouin zone of the lattice defined by the proper translations $\mathbf{t}_{1}^{\prime}$ and $\mathbf{t}_{2}^{\prime}$ ) are realized by the following linear combinations:

$$
\begin{aligned}
& \left|\Psi_{2, \mathbf{0}}^{0}\right\rangle=\frac{1}{\sqrt{N}}\left(\sum_{j \in A}\left|\Psi_{2}^{0}(l)\right\rangle+C_{2}^{\|} \sum_{j \in B}\left|\Psi_{2}^{0}(l)\right\rangle\right), \\
& \left|\Psi_{2, \boldsymbol{\pi}}^{0}\right\rangle=\frac{1}{\sqrt{N}}\left(\sum_{j \in A}\left|\Psi_{2}^{0}(l)\right\rangle-C_{2}^{\|} \sum_{j \in B}\left|\Psi_{2}^{0}(l)\right\rangle\right) .
\end{aligned}
$$

Then, the energies of the excitations are

$$
\begin{aligned}
\omega_{\mathbf{k}}^{1 \rightarrow 2} & =E_{\mathbf{k}, 2}^{(1)}-E^{(1)} \\
& =\left\langle\Psi_{2, \mathbf{k}}^{0}|\mathcal{H}| \Psi_{2, \mathbf{k}}^{0}\right\rangle-\left\langle\Psi_{1}^{0}|\mathcal{H}| \Psi_{1}^{0}\right\rangle .
\end{aligned}
$$

The evaluation of the expectation values using the single-ion wave functions (B2) is straightforward but tedious.

Keeping only the first-order terms of the perturbation and doing series expansion we get, in the strong magnetic field limit $h \gg \Lambda \gg J, J_{z}$, and $D_{z}=0$,

$$
\begin{aligned}
& \omega_{0}^{1 \rightarrow 2}=h+\Lambda-9 J-3 J_{z}, \\
& \omega_{\pi}^{1 \rightarrow 2}=h+\Lambda-3 J+3 J_{z} .
\end{aligned}
$$


The perturbative approach works well for the experimentally studied paramagnetic case in the high-field limit. In Sec. VII A 2 we have seen that only the $\pi$ modes absorb in Faraday geometry. In the exact diagonalization, we observed the strongest absorption is for the $\omega_{\pi}^{1 \rightarrow 2}$ mode. This is not surprising, as this is the single-magnon mode in the weak anisotropy limit $\Lambda \ll J, J_{z}$. In the single-ion model without anisotropy $J, J_{z}, \Lambda=0$, it is just the paramagnetic mode with $\omega_{\pi}^{1 \rightarrow 2}=h$, as it can be inferred from Eq. (37b). More interestingly, the energy of this mode is very close to the single-ion excitation energy $\omega^{1 \rightarrow 2}=\varepsilon_{2}-\varepsilon_{1}$ [see Eq. (B6)]. The difference is just $3\left(J-J_{z}\right)$ and vanishes for $J=J_{z}$. This explains why the single-ion model works remarkably well for large magnetic fields $h \gg \Lambda, J, J_{z}$, as seen from the comparison of the minimal theory and the experiments in Fig. 6.

In the strong single-ion anisotropy limit $\Lambda \gg h, J, J_{z}$, the energies of these modes are

$$
\begin{aligned}
& \omega_{0}^{1 \rightarrow 2}=2 h-12 J-J_{z}, \\
& \omega_{\pi}^{1 \rightarrow 2}=2 h-4 J+J_{z} .
\end{aligned}
$$

The deviation of $\omega_{\pi}^{1 \rightarrow 2}$ in the single-ion model becomes noticeable compared to the strong field limit given by Eq. (37b), $-4 J+J_{z}$ vs $-3 J+3 J_{z}$, but still not too large as compared to the deviations of other modes, say Eq. (38a).

\section{SUMMARY}

The coupling of the magnetic moments and electric polarization in a material is responsible for many interesting optical phenomena which happen at low temperatures, usually in the ordered phases. Here, we studied the optical response of a multiferroic material in the paramagnetic phase, at temperatures much higher than the ordering temperature.

We observed MChD for the excitations of the $S=\frac{3}{2}$ spin of the $\mathrm{Co}^{2+}$ ion in $\mathrm{Sr}_{2} \mathrm{CoSi}_{2} \mathrm{O}_{7}$ at temperatures up to $100 \mathrm{~K}$, although the magnetic ordering temperature is only $T_{\mathrm{N}}=7 \mathrm{~K}$. The main experimental findings, the temperature dependence of the spin-mode frequencies, their intensities, and the sign of the MChD for the different spin modes, are captured well by the exact diagonalization of a four-spin cluster.

The numerical results are interpreted in a simple analytical model of a single spin with an easy-plane anisotropy in an external magnetic field, and its coupling to the $\mathrm{THz}$ radiation via magnetization $\mathbf{M}$ and via polarization $\mathbf{P}$, expressed by spin-quadrupolar terms. Finite ME susceptibility arises if the components of the $\mathbf{M}$ and $\mathbf{P}$ transform according to the same irreducible representation of the symmetry group compatible with both, the oxygen tetrahedron around the magnetic ion and the applied magnetic field. Figure 6 shows that in high fields and low temperatures $\left(\varepsilon_{2}-\varepsilon_{1}>k_{\mathrm{B}} T\right)$ the NDD spectrum is dominated by the magnetic dipolar transition from the ground state $|1\rangle \rightarrow|2\rangle$. By increasing $T$, another magnetic dipolar transition from the thermally excited state $|2\rangle \rightarrow|3\rangle$ appears in the NDD spectrum. Our exact diagonalization calculation showed that the $|2\rangle \rightarrow|3\rangle$ peak is very close to the $|1\rangle \rightarrow|2\rangle$ in energy and due to the line-broadening effects only a single broad peak is observed. This coincides with the experimental finding at high temperatures, as exemplified in Figs. 2(a) and 2(b).

By considering the real material where the the oxygen tetrahedra are tilted, we showed how the selection rules of the single-ion model are modified when the exchange coupling is turned on, in agreement with the results of the exact diagonalization. Finally, we demonstrated that the exchange correlations are important to accurately describe the mode frequencies in the paramagnetic state.

In conclusion, we demonstrated that $\mathrm{MChD}$ can arise in the paramagnetic phase of a noncentrosymmetric material. Furthermore, we presented a detailed theoretical analysis of spin excitations in $\mathrm{Sr}_{2} \mathrm{CoSi}_{2} \mathrm{O}_{7}$ which helps to identify the key parameters responsible for high-temperature NDD both in the chiral and toroidal cases.

\section{ACKNOWLEDGMENTS}

This research was supported by the Estonian Ministry of Education and Research with institutional research funding IUT23-3, by the European Regional Development Fund Project No. TK134, by the bilateral program of the Estonian and Hungarian Academies of Sciences under the Contract No. SNK-64/2013, by the Hungarian NKFIH Grants No. K 124176 and No. ANN 122879, by the BMENanonotechnology and Materials Science FIKP grant of EMMI (BME FIKP-NAT), by the FWF Austrian Science Fund No. I 2816-N27, and by the Deutsche Forschungsgemeinschaft (DFG) via the Transregional Research Collaboration TRR 80: From Electronic Correlations to Functionality (Augsburg-Munich-Stuttgart) We acknowledge the support of the HFML-RU/FOM, member of the European Magnetic Field Laboratory (EMFL). V.K. gratefully acknowledges support from RIKEN's FY 2016 Incentive Research Projects.

V.K. and Y. Tokunaga grew the crystals, D.G.F., D.Sz., V.K., J.V. and U.N. conducted THz spectroscopy experiments in Tallinn and analyzed the results, D.G.F., D.Sz., B.B. and D.L.K. conducted high magnetic field measurement in Nijmegen, P.B. and K.P. developed the theory, T.R., S.B., P.B., and K.P. wrote the manuscript, D.Sz. and K.P. conceived the project. Every author contributed to the discussion of the results.

\section{APPENDIX A: REALITY OF THE MATRIX ELEMENTS IN A MAGNETIC POINT GROUP}

We analyze the case of $\varphi=0$, which is relevant for the experimental situation and is conceptually the simplest. The symmetry group of this configuration considering both the spin Hamiltonian (14) and the oxygen tetrahedron around the $\mathrm{Co}^{2+}$ ion, manifested by the spin-polarization coupling (15), is

$$
D_{2}\left(C_{2}\right)=\left\{\mathbf{1}, C_{2}^{\|}, \Theta C_{2}^{\perp 1}, \Theta C_{2}^{\perp 2}\right\},
$$

with operators in the field-fixed coordinate frame (Fig. 5). Since the inversion, the mirror plane, and the rotoreflection symmetry are absent, the case is termed as chiral. The $D_{2}\left(C_{2}\right)$ group is generated by the symmetry elements $C_{2}^{\|}$and $\Theta C_{2}^{\perp 2}$, and its character table together with the symmetry classification of spin and polarization components is summarized 
in Table III. We note that our conclusions are equally valid for the $\varphi=\pi / 4$ case, where $\Theta C_{2}^{\perp 2}$ is also present in the corresponding polar symmetry group $C_{2 v}\left(C_{1 h}\right)$.

The operator $C_{2}^{\perp 2}$ is represented on the $S=\frac{3}{2}$ spin space by the matrix

$$
\hat{C}_{2}^{\perp 2}=e^{i \pi \hat{S}^{\perp 2}}=\left(\begin{array}{cccc}
0 & 0 & 0 & 1 \\
0 & 0 & -1 & 0 \\
0 & 1 & 0 & 0 \\
-1 & 0 & 0 & 0
\end{array}\right) .
$$

The time-reversal operation $\Theta$ is conveniently represented by the antiunitary operation

$$
\hat{\Theta}=\hat{C}_{2}^{\perp 2} \mathcal{K}=e^{i \pi \hat{S}^{\perp 2}} \mathcal{K}=\left(\begin{array}{cccc}
0 & 0 & 0 & 1 \\
0 & 0 & -1 & 0 \\
0 & 1 & 0 & 0 \\
-1 & 0 & 0 & 0
\end{array}\right) \mathcal{K},
$$

where $\mathcal{K}$ is the complex conjugation, conjugating every matrix or vector component on the right, but leaving the basis functions intact [31]. This is analogous to the $S=\frac{1}{2}$ case where $\Theta$ is expressed with the help of the Pauli matrix $\hat{\sigma}^{y}$ as $\hat{\Theta}=i \hat{\sigma}^{y} \mathcal{K}$. Collecting all this together, the operator for $\Theta C_{2}^{\perp 2}$ in the Hilbert space reads as

$$
\hat{\Theta} \hat{C}_{2}^{\perp 2}=\hat{C}_{2}^{\perp 2} \mathcal{K} \hat{C}_{2}^{\perp 2}=e^{i 2 \pi \hat{S}^{\perp 2}} \mathcal{K}=-\hat{\mathbf{1}} \mathcal{K} .
$$

When we consider the effect of this symmetry on the matrix elements of physical observables, we find first all representations of $\Theta C_{2}^{\perp 2}$ are +1 or -1 as given in Table III for the chiral and polar cases, respectively. Therefore, any linear operator $\mathcal{O}$, such as the spin or polarization components, is either even or odd, i.e., it transforms as

$$
\Theta C_{2}^{\perp 2}(\mathcal{O})= \pm \mathcal{O}
$$

or in matrix representation

$$
\left(\hat{\Theta} \hat{C}_{2}^{\perp 2}\right) \hat{\mathcal{O}}\left(\hat{\Theta} \hat{C}_{2}^{\perp 2}\right)^{-1}= \pm \hat{\mathcal{O}} .
$$

Next, following Eq. (A4), let us evaluate the left-hand side of the equation above:

$$
\left(\hat{\Theta} \hat{C}_{2}^{\perp 2}\right) \hat{\mathcal{O}}\left(\hat{\Theta} \hat{C}_{2}^{\perp 2}\right)^{-1}=\mathcal{K} \hat{\mathcal{O}} \mathcal{K}=\hat{\mathcal{O}}^{*},
$$

where the last equality follows from the fact that acting on an arbitrary vector $v, \mathcal{K} \hat{\mathcal{O}} \mathcal{K} v=\mathcal{K} \hat{\mathcal{O}} v^{*}=\hat{\mathcal{O}}^{*} v$. Equations (A6) and (A7) together mean that

$$
\hat{\mathcal{O}}^{*}= \pm \hat{\mathcal{O}} .
$$

This restricts the matrix elements of the operators even under $\Theta C_{2}^{\perp 2}$ to be real, and the matrix elements of the odd operators to be pure imaginary.

In conclusion, the unitary symmetries determine the selection rules, i.e., the nonvanishing matrix elements, whereas antiunitary elements give constraints on the reality of them. These rules and the matrix elements given in Appendix B (and calculated by using the explicit wave functions) are in full agreement.

\section{APPENDIX B: SOLUTION OF THE SINGLE-ION PROBLEM}

\section{Energies and wave functions}

The energies in increasing order are

$$
\begin{aligned}
& \varepsilon_{1}^{(-i)}=-\frac{h}{2}+\frac{5 \Lambda}{4}-\sqrt{h^{2}+h \Lambda+\Lambda^{2}}, \\
& \varepsilon_{2}^{(+i)}=\frac{h}{2}+\frac{5 \Lambda}{4}-\sqrt{h^{2}-h \Lambda+\Lambda^{2}}, \\
& \varepsilon_{3}^{(-i)}=-\frac{h}{2}+\frac{5 \Lambda}{4}+\sqrt{h^{2}+h \Lambda+\Lambda^{2}}, \\
& \varepsilon_{4}^{(+i)}=\frac{h}{2}+\frac{5 \Lambda}{4}+\sqrt{h^{2}-g h \Lambda+\Lambda^{2}},
\end{aligned}
$$

and the corresponding unnormalized eigenstates are

$$
\begin{aligned}
& \left|\psi_{1}^{(-i)}\right\rangle \propto\left(2 h+\Lambda+2 \sqrt{h^{2}+h \Lambda+\Lambda^{2}}\right)|\Uparrow\rangle+\sqrt{3} \Lambda|\downarrow\rangle, \\
& \left|\psi_{2}^{(+i)}\right\rangle \propto\left(2 h-\Lambda+2 \sqrt{h^{2}-h \Lambda+\Lambda^{2}}\right)|\uparrow\rangle+\sqrt{3} \Lambda|\Downarrow\rangle, \\
& \left|\psi_{3}^{(-i)}\right\rangle \propto\left(2 h+\Lambda+2 \sqrt{h^{2}+h \Lambda+\Lambda^{2}}\right)|\downarrow\rangle-\sqrt{3} \Lambda|\Uparrow\rangle, \\
& \left|\psi_{4}^{(+i)}\right\rangle \propto\left(2 h-\Lambda+2 \sqrt{h^{2}-h \Lambda+\Lambda^{2}}\right)|\Downarrow\rangle-\sqrt{3} \Lambda|\uparrow\rangle .
\end{aligned}
$$

The phases for the eigenvectors above are chosen in such a way that we recover the basis $\{|\uparrow\rangle,|\uparrow\rangle,|\downarrow\rangle,|\Downarrow\rangle\}$ for $h \gg \Lambda$, e.g., $\left|\psi_{1}^{(-i)}\right\rangle \rightarrow|\Uparrow\rangle$, and so on.

\section{Large anisotropy and small field: $\Lambda \gg h$}

From the series expansion of the single-spin energies (B1) in $h / \Lambda$, we get

$$
\begin{aligned}
& \varepsilon_{1}=\frac{\Lambda}{4}-h-\frac{3 h^{2}}{8 \Lambda}+\cdots, \\
& \varepsilon_{2}=\frac{\Lambda}{4}+h-\frac{3 h^{2}}{8 \Lambda}+\cdots, \\
& \varepsilon_{3}=\frac{9 \Lambda}{4}+\frac{3 h^{2}}{8 \Lambda}+\cdots, \\
& \varepsilon_{4}=\frac{9 \Lambda}{4}+\frac{3 h^{2}}{8 \Lambda}+\cdots .
\end{aligned}
$$

The spin and polarization operators in leading order of $h / \Lambda$ are in the matrix representation

$$
\hat{S}^{\|}=\left(\begin{array}{cccc}
1 & 0 & -\frac{\sqrt{3}}{2} & 0 \\
0 & -1 & 0 & -\frac{\sqrt{3}}{2} \\
-\frac{\sqrt{3}}{2} & 0 & \frac{9 h^{2}}{16 \Lambda^{2}} & 0 \\
0 & -\frac{\sqrt{3}}{2} & 0 & -\frac{9 h^{2}}{16 \Lambda^{2}}
\end{array}\right) \text {, }
$$




$$
\begin{aligned}
& \hat{S}^{\perp 1}=\left(\begin{array}{cccc}
0 & 1 & 0 & -\frac{\sqrt{3}}{2} \\
1 & 0 & \frac{\sqrt{3}}{2} & 0 \\
0 & \frac{\sqrt{3}}{2} & 0 & \frac{9 h^{2}}{16 \Lambda^{2}} \\
-\frac{\sqrt{3}}{2} & 0 & \frac{9 h^{2}}{16 \Lambda^{2}} & 0
\end{array}\right), \\
& \hat{S}^{\perp 2}=i\left(\begin{array}{cccc}
0 & -\frac{1}{2} & 0 & \frac{\sqrt{3} h}{4 \Lambda} \\
\frac{1}{2} & 0 & -\frac{\sqrt{3} h}{4 \Lambda} & 0 \\
0 & \frac{\sqrt{3} h}{4 \Lambda} & 0 & -\frac{3}{2} \\
-\frac{\sqrt{3} h}{4 \Lambda} & 0 & \frac{3}{2} & 0
\end{array}\right) \text {, } \\
& \hat{P}_{\text {chiral }}^{\|}=-\hat{P}_{\text {polar }}^{\perp 1}=i\left(\begin{array}{cccc}
0 & 0 & -\sqrt{3} & 0 \\
0 & 0 & 0 & -\sqrt{3} \\
\sqrt{3} & 0 & 0 & 0 \\
0 & \sqrt{3} & 0 & 0
\end{array}\right) \text {, } \\
& \hat{P}_{\text {polar }}^{\|}=\hat{P}_{\text {chiral }}^{\perp 1}=i\left(\begin{array}{cccc}
0 & -\frac{3 h}{2 \Lambda} & 0 & \sqrt{3} \\
\frac{3 h}{2 \Lambda} & 0 & -\sqrt{3} & 0 \\
0 & \sqrt{3} & 0 & \frac{3 h}{2 \Lambda} \\
-\sqrt{3} & 0 & -\frac{3 h}{2 \Lambda} & 0
\end{array}\right) \text {, } \\
& \hat{P}_{\text {chiral }}^{\perp 2}=\left(\begin{array}{cccc}
0 & \frac{3 h}{2 \Lambda} & 0 & -\sqrt{3} \\
\frac{3 h}{2 \Lambda} & 0 & -\sqrt{3} & 0 \\
0 & -\sqrt{3} & 0 & -\frac{3 h}{2 \Lambda} \\
-\sqrt{3} & 0 & -\frac{3 h}{2 \Lambda} & 0
\end{array}\right), \\
& \hat{P}_{\text {polar }}^{\perp 2}=\left(\begin{array}{cccc}
\frac{3 h}{2 \Lambda} & 0 & -\sqrt{3} & 0 \\
0 & -\frac{3 h}{2 \Lambda} & 0 & \sqrt{3} \\
-\sqrt{3} & 0 & -\frac{3 h}{2 \Lambda} & 0 \\
0 & \sqrt{3} & 0 & \frac{3 h}{2 \Lambda}
\end{array}\right) \text {. }
\end{aligned}
$$

\section{Large field and small anisotropy: $h \gg \Lambda$}

The energies are

$$
\begin{aligned}
& \varepsilon_{1}=-\frac{3 h}{2}+\frac{3 \Lambda}{4}-\frac{3 \Lambda^{2}}{8 h}+\cdots, \\
& \varepsilon_{2}=-\frac{h}{2}+\frac{7 \Lambda}{4}-\frac{3 \Lambda^{2}}{8 h}+\cdots, \\
& \varepsilon_{3}=\frac{h}{2}+\frac{7 \Lambda}{4}+\frac{3 \Lambda^{2}}{8 h}+\cdots,
\end{aligned}
$$

$$
\varepsilon_{4}=\frac{3 h}{2}+\frac{3 \Lambda}{4}+\frac{3 \Lambda^{2}}{8 h}+\cdots
$$

(B4b) The spin and polarization operators in the leading order of $\Lambda / h$ are in the matrix representation

$$
\begin{aligned}
& \hat{S}^{\|}=\left(\begin{array}{cccc}
\frac{3}{2} & 0 & -\frac{\sqrt{3} \Lambda}{2 h} & 0 \\
0 & \frac{1}{2} & 0 & -\frac{\sqrt{3} \Lambda}{2 h} \\
-\frac{\sqrt{3} \Lambda}{2 h} & 0 & -\frac{1}{2} & 0 \\
0 & -\frac{\sqrt{3} \Lambda}{2 h} & 0 & -\frac{3}{2}
\end{array}\right), \\
& \hat{S}^{\perp 1}=\left(\begin{array}{cccc}
0 & \frac{\sqrt{3}}{2} & 0 & -\frac{9 \Lambda^{2}}{16 h^{2}} \\
\frac{\sqrt{3}}{2} & 0 & 1 & 0 \\
0 & 1 & 0 & \frac{\sqrt{3}}{2} \\
-\frac{9 \Lambda^{2}}{16 h^{2}} & 0 & \frac{\sqrt{3}}{2} & 0
\end{array}\right), \\
& \hat{S}^{\perp 2}=i\left(\begin{array}{cccc}
0 & -\frac{\sqrt{3}}{2} & 0 & \frac{3 \Lambda^{2}}{16 h^{2}} \\
\frac{\sqrt{3}}{2} & 0 & -1 & 0 \\
0 & 1 & 0 & -\frac{\sqrt{3}}{2} \\
-\frac{3 \Lambda^{2}}{16 h^{2}} & 0 & \frac{\sqrt{3}}{2} & 0
\end{array}\right) \text {, } \\
& \hat{P}_{\text {chiral }}^{\|}=-\hat{P}_{\text {polar }}^{\perp 1}=i\left(\begin{array}{cccc}
0 & 0 & -\sqrt{3} & 0 \\
0 & 0 & 0 & -\sqrt{3} \\
\sqrt{3} & 0 & 0 & 0 \\
0 & \sqrt{3} & 0 & 0
\end{array}\right) \text {, } \\
& \hat{P}_{\text {polar }}^{\|}=\hat{P}_{\text {chiral }}^{\perp 1}=i\left(\begin{array}{cccc}
0 & -\sqrt{3} & 0 & \frac{3 \Lambda}{2 h} \\
\sqrt{3} & 0 & -\frac{3 \Lambda}{2 h} & 0 \\
0 & \frac{3 \Lambda}{2 h} & 0 & \sqrt{3} \\
-\frac{3 \Lambda}{2 h} & 0 & -\sqrt{3} & 0
\end{array}\right) \text {, } \\
& \hat{P}_{\text {chiral }}^{\perp 2}=\left(\begin{array}{cccc}
0 & \sqrt{3} & 0 & -\frac{3 \Lambda}{2 h} \\
\sqrt{3} & 0 & -\frac{3 \Lambda}{2 h} & 0 \\
0 & -\frac{3 \Lambda}{2 h} & 0 & -\sqrt{3} \\
-\frac{3 \Lambda}{2 h} & 0 & -\sqrt{3} & 0
\end{array}\right) \text {, } \\
& \hat{P}_{\text {polar }}^{\perp 2}=\left(\begin{array}{cccc}
\frac{3}{2} & 0 & -\frac{\sqrt{3}}{2} & 0 \\
0 & -\frac{3}{2} & 0 & -\frac{\sqrt{3}}{2} \\
-\frac{\sqrt{3}}{2} & 0 & -\frac{3}{2} & 0 \\
0 & -\frac{\sqrt{3}}{2} & 0 & \frac{3}{2}
\end{array}\right) \text {. }
\end{aligned}
$$

[6] I. Kézsmárki, N. Kida, H. Murakawa, S. Bordács, Y. Onose, and Y. Tokura, Phys. Rev. Lett. 106, 057403 (2011).

[7] S. Bordács, I. Kézsmárki, D. Szaller, L. Demkó, N. Kida, H. Murakawa, Y. Onose, R. Shimano, T. Rõõm, U. Nagel, S. Miyahara, N. Furukawa, and Y. Tokura, Nat. Phys. 8, 734 (2012).

[8] Y. Takahashi, R. Shimano, Y. Kaneko, H. Murakawa, and Y. Tokura, Nat. Phys. 8, 121 (2012). 
[9] Y. Takahashi, Y. Yamasaki, and Y. Tokura, Phys. Rev. Lett. 111, 037204 (2013).

[10] I. Kézsmárki, D. Szaller, S. Bordács, V. Kocsis, Y. Tokunaga, Y. Taguchi, H. Murakawa, Y. Tokura, H. Engelkamp, T. Rõõm, and U. Nagel, Nat. Commun. 5, 3203 (2014).

[11] A. M. Kuzmenko, V. Dziom, A. Shuvaev, A. Pimenov, M. Schiebl, A. A. Mukhin, V. Y. Ivanov, I. A. Gudim, L. N. Bezmaternykh, and A. Pimenov, Phys. Rev. B 92, 184409 (2015).

[12] I. Kézsmárki, U. Nagel, S. Bordács, R. S. Fishman, J. H. Lee, H. T. Yi, S.-W. Cheong, and T. Rõõm, Phys. Rev. Lett. 115, 127203 (2015).

[13] D. Szaller, S. Bordács, V. Kocsis, T. Rõõm, U. Nagel, and I. Kézsmárki, Phys. Rev. B 89, 184419 (2014).

[14] A. M. Kuzmenko, D. Szaller, T. Kain, V. Dziom, L. Weymann, A. Shuvaev, A. Pimenov, A. A. Mukhin, V. Y. Ivanov, I. A. Gudim, L. N. Bezmaternykh, and A. Pimenov, Phys. Rev. Lett. 120, 027203 (2018).

[15] V. Kocsis, K. Penc, T. Rõõm, U. Nagel, J. Vít, J. Romhányi, Y. Tokunaga, Y. Taguchi, Y. Tokura, I. Kézsmárki, and S. Bordács, Phys. Rev. Lett. 121, 057601 (2018).

[16] M. Akaki, H. Iwamoto, T. Kihara, M. Tokunaga, and H. Kuwahara, Phys. Rev. B 86, 060413 (2012).

[17] M. Akaki, T. Tadokoro, T. Kihara, M. Tokunaga, and H. Kuwahara, J. Low Temp. Phys. 170, 291 (2013).

[18] S. Yu, B. Gao, J. W. Kim, S.-W. Cheong, M. K. L. Man, J. Madéo, K. M. Dani, and D. Talbayev, Phys. Rev. Lett. 120, 037601 (2018).

[19] H. Murakawa, Y. Onose, S. Miyahara, N. Furukawa, and Y. Tokura, Phys. Rev. Lett. 105, 137202 (2010).

[20] K. Penc, J. Romhányi, T. Rõõm, U. Nagel, A. Antal, T. Fehér, A. Jánossy, H. Engelkamp, H. Murakawa, Y. Tokura, D. Szaller, S. Bordács, and I. Kézsmárki, Phys. Rev. Lett. 108, 257203 (2012).

[21] M. Soda, M. Matsumoto, M. Månsson, S. Ohira-Kawamura, K. Nakajima, R. Shiina, and T. Masuda, Phys. Rev. Lett. 112, 127205 (2014).

[22] J. Romhányi, M. Lajkó, and K. Penc, Phys. Rev. B 84, 224419 (2011).
[23] T. Arima, J. Phys. Soc. Jpn. 76, 073702 (2007).

[24] S. Miyahara and N. Furukawa, J. Phys. Soc. Jpn. 80, 073708 (2011).

[25] K. Yamauchi, P. Barone, and S. Picozzi, Phys. Rev. B 84, 165137 (2011).

[26] The absorption is given by

$$
\alpha^{ \pm}(\omega)=\frac{2 \omega}{c_{0}} \operatorname{Im} \mathcal{N}_{ \pm}(\omega)
$$

where the complex index of refraction $\mathcal{N}_{ \pm}$for the $\pm k$ direction of light propagation is

$$
\mathcal{N}_{ \pm}(\omega)=\sqrt{\left[\epsilon_{\nu \nu}+\chi_{\nu \nu}^{e e}(\omega)\right]\left[\mu_{\mu \mu}+\chi_{\mu \mu}^{m m}(\omega)\right]} \pm \chi_{\mu \nu}^{m e}(\omega),
$$

where $\chi_{\mu \nu}^{m e}(\omega)$ is the time-reversal odd part of the ME susceptibility. $\epsilon_{v v}$ and $\mu_{\mu \mu}$ are real background dielectric permittivity and magnetic permeability, respectively, not originating from the spin system. In the limit of a small spin contribution to the susceptibilities, $\chi_{v v}^{e e}(\omega) \ll \epsilon_{v v}, \chi_{\mu \mu}^{m m}(\omega) \ll \mu_{\mu \mu}$, index of refraction is

$$
\begin{aligned}
\mathcal{N}_{ \pm}(\omega) \approx & \sqrt{\epsilon_{v \nu} \mu_{\mu \mu}}+\frac{\sqrt{\mu_{\mu \mu}}}{2 \sqrt{\epsilon_{v \nu}}} \chi_{\nu v}^{e e}(\omega) \\
& +\frac{\sqrt{\epsilon_{\nu \nu}}}{2 \sqrt{\mu_{\mu \mu}}} \chi_{\mu \mu}^{m m}(\omega) \pm \chi_{\mu \nu}^{m e}(\omega) .
\end{aligned}
$$

[27] Since the $S=\frac{3}{2}$ has four states, the dimension of the Hilbert space of the four-spin cluster is $4^{4}=256$, and the sums in Eqs. (8) and (9) run over $256^{2}=65536$ terms. Even though the cluster is small, it already provided sufficient information to compare with the experimental data at high temperatures and fields.

[28] L. Barron and J. Vrbancich, Mol. Phys. 51, 715 (1984).

[29] M. Akaki, D. Yoshizawa, A. Okutani, T. Kida, J. Romhányi, K. Penc, and M. Hagiwara, Phys. Rev. B 96, 214406 (2017).

[30] S. Altmann and P. Herzig, Point-group Theory Tables, Oxford Science Publications (Clarendon, Oxford, 1994).

[31] J. Sakurai and J. Napolitano, Modern Quantum Mechanics (Addison-Wesley, Boston, 2011). 